\title{
Strukturelle Gewalt - ein analytisch überschätzter Begriff
}

\author{
Andreas Braun (iD
}

Eingegangen: 7. Oktober 2019 / Überarbeitet: 10. Januar 2021 / Angenommen: 6. Februar 2021 / Online publiziert: 26. März 2021

(C) Der/die Autor(en) 2021

Zusammenfassung Der Beitrag widmet sich vor dem Hintergrund der jüngst von Peter Imbusch vorgeschlagenen Revitalisierung dem Konzept der strukturellen Gewalt. Entgegen der von Imbusch vertretenen fruchtbaren Reintegration des Konzeptes in neuere gewaltsoziologische Forschungen wird hier die These einer diesbezüglichen analytischen Überschätzung vertreten. Vor dem Hintergrund eines einfachen (bezugsproblemorientierten) Vergleichs wird aufgezeigt, dass eine Berücksichtigung für die neuere gewaltsoziologische Forschung insofern überschätzt wird, als das Konzept nicht auf Gewalt (violentia), sondern auf Macht (potestas) abzielt. Darüber hinaus wird konstatiert, dass die von Imbusch postulierte gewaltsoziologische Engführung auf Körper und Leid keineswegs eine nicht zu rechtfertigende Fokussierung darstellt. Gerade Ansätze der neueren gewaltsoziologischen Forschung sind mit diesem Bezugsrahmen in der Lage, Gewalt und Macht analytisch trennscharf zu behandeln und eine differenzierte Betrachtung struktureller und kultureller Faktoren im Kontext nicht normativer und a priori dyadischer (de-)legitimierender Gewaltbeobachtungen vorzunehmen. Aspekte, die sich hingegen für eine galtungsche Friedensund Konfliktforschung als fruchtbar erweisen können.

Schlüsselwörter Gewalt (violentia) · Macht (potestas) · Bezugsproblem · Abgrenzungsprobleme $\cdot($ Selbst-)Beobachtung $\cdot($ De-)Legitimation

\footnotetext{
A. Braun $(\bowtie)$

Medizinische Fakultät OWL, Universität Bielefeld, Universitätsstraße 25, 33615 Bielefeld, Deutschland

E-Mail: andreas.braun@uni-bielefeld.de
} 


\title{
Structural violence—an analytically overrated term
}

\begin{abstract}
Against the background of the recently proposed fruitful revitalization of Galtung's approach into the sociological violence research by Peter Imbusch, this article focus on structural violence. Contrary to Imbusch, here, the thesis of an analytical overestimation of structural violence is presented. Given the fact of a simple (reference problem-oriented) comparison, structural violence is insofar overestimating for the recent sociological violence research as it does not aim at violence (violentia) but power (potestas). Moreover, the sociological focus on body and suffering as postulated by Imbusch is by no means unjustifiable. With this frame of reference, sociological violence research can treat violence and power with a high degree of analytical precision and conduct a differentiated examination of structural and cultural factors in the context of non-normative and a priori dyadic (de-)legitimizing observations of violence. These aspects, on the other hand, can prove fruitful for constructive peace and conflict research.
\end{abstract}

Keywords Violence (violentia) · Power (potestas) · Problem of reference · Problem of delimitation · (Self-)observation · (De-)legitimation

\section{Einleitung}

Das Konzept der strukturellen Gewalt, das 1969 von Johan Galtung geprägt wurde, zählt wohl ohne Zweifel zu einem Klassiker der Friedens- und Konfliktforschung. Während Galtungs Theorie und sein hiermit verbundenes Verständnis von Gewalt in unterschiedlichen (interdisziplinären) Forschungskontexten durchaus fruchtbar rezipiert, integriert und auch aktualisiert wurde, war und ist dessen Einführung in die generelle Gewaltdiskussion stets Bestandteil einer kritischen Auseinandersetzung. ${ }^{1}$ Nicht zuletzt im Zuge der aufkommenden neuen Gewaltsoziologie gegen Ende des 20. Jahrhunderts im deutschsprachigen Kontext, die mit dem konträr zur damals vorherrschenden Gewaltforschung stehenden Label der „Innovateure“ (Imbusch 2004) gekennzeichnet ist, führte eine Engfassung des Gewaltbegriffs auf explizite physische Gewalt und damit verbundenen Leids zu einer Ablehnung struktureller Gewalt. ${ }^{2}$ Diese resultierte vor allem aus einem hiermit verbundenen zu weit gefassten Gewaltverständnis, welches letztlich jegliche Erscheinung als Gewalt beschreibbar werden lässt, die Gefahr einer drohenden - Gewalt durch Gewalt erklärenden - Tautologie birgt und somit in eine inflationäre Bedeutung von Gewalt mündet (vgl. Imbusch 2017a, S. 31-35; Knöbl 2019, S. 35; Schinkel 2010, S. 39). ${ }^{3}$

\footnotetext{
1 Vgl. zur fruchtbaren Rezeption zusammenfassend Imbusch (2017a, Fn. 26-27) sowie u.a. Brunner (2020); De Maio und Ansell (2018); Hamer und Lang (2015); Hodgetts et al. (2013); Schmidt (2001) und zur gewaltsoziologischen Kritik zusammenfassend Imbusch (2017a, S. 31-36).

2 Vgl. Baecker (2007, S. 36-38); Baberowski (2008, S. 7-9); Imbusch (2004); Nedelmann (1997, S. 61); Nunner-Winkler (2004, S. 23, 45-46); Riekenberg (2008); von Trotha (1997, S. 14).

3 Obgleich sich die folgenden Ausführungen explizit auf den deutschsprachigen Forschungskontext beziehen, was sich durch die spezifisch geprägte Diskussionshistorie und den nachfolgend im Fokus stehenden Reintegrationsvorschlag struktureller Gewalt durch Peter Imbusch (2017a, S. 37-42) begründet, welcher
} 
Auch einige Dekaden nach dem gewaltsoziologischen Diskurs zwischen „,Mainstreamer[n] versus ,Innovateure[n] ““ (Imbusch 2004) nimmt das Konzept der strukturellen Gewalt in neueren Ansätzen der Gewaltforschung, die sich auf dynamische Gewaltsituationen, prozessual verlaufende Gewaltereignisse sowie auf Reflexivität und variierende Ordnungen von Gewalt fokussieren, keinen Stellenwert ein (vgl. Braun 2020; Equit und Schmidt 2016, S. 49; Hauffe und Hoebel 2017). Strukturelle Gewalt scheint demnach weiterhin mehr als Konzept zur Abgrenzung einer neueren Gewaltforschung zu fungieren denn als kritisch $\mathrm{zu}$ reflektierendes ernsthaftes Pendant (vgl. Imbusch 2017a, S. 31-36).

Vor diesem Hintergrund hat Peter Imbusch (2017a) jüngst die Wiederbelebung der Diskussion um und für das galtungsche (1969, 1975a, 1990, 1993b) Konzept unternommen. Gerade mit Blick auf die potenzielle Erweiterung der aktuellen gewaltsoziologischen Forschung um Aspekte sozialstruktureller bzw. sozio-kultureller Wirkfaktoren oder zur Sichtbarmachung gesellschaftlich verankerter und Gewalt produzierender Problemlagen sei Galtungs Konzept nicht nur für eine grundlegend kritisch angelegte Gesellschaftsanalyse fruchtbar, sondern auch für die neuere soziologische Gewaltforschung (vgl. Imbusch 2017a, S. 37-50). Imbuschs (2017a) Vorschlag einer gewaltsoziologischen Reintegration struktureller Gewalt, der im Fokus der nachfolgenden Überlegungen steht, scheint dabei auf den ersten Blick erhellend zu sein. Bedenkt man doch diesbezüglich z. B. jüngere gewaltsoziologische Ansätze, die den Blick ebenso auf nicht sichtbare Gewalt und ihre Indexikalität sowie auf soziale Hintergrund- und Kontextfaktoren richten und diesen als Gewalt begünstigende Faktoren und Elemente Bedeutung zusprechen. ${ }^{4}$

So plausibel die vorgeschlagene Reintegration in aktuelle Gewaltforschungen auf den ersten Blick scheint, so muss in kritischer Hinsicht aber dennoch die Frage nach dem analytischen Mehrwert des vorgetragenen Plädoyers und eines erweiterten Gewaltbegriffs gestellt werden (vgl. Knöbl 2019, S. 32-34). Schließlich existieren die grundlegenden Schwachstellen, aufgrund derer ,,sich das ursprüngliche Konzept der strukturellen Gewalt [...] nur schwerlich revitalisieren ließe“ (Imbusch 2017a, S. 37), weiterhin (vgl. Imbusch 2017a, S. 37; Knöbl 2019, S. 32-34). Im Folgenden soll demnach die von Imbusch (2017a, S. 37) aufgeworfene Frage, ob ,man also die Galtung'sche Begriffsschöpfung in Bausch und Bogen verwerfen [muss]", aus einer explizit gewaltsoziologischen Perspektive kritisch beleuchtet werden.

Die These lautet hierbei, dass strukturelle Gewalt gerade aufgrund ihres zentralen Machtbezuges keinen analytisch gewinnbringenden Anschluss an neuere gewaltsoziologische Ansätze ermöglicht, da diese auf körperlichen ${ }^{5}$ Akteur*innen basieren-

diesen ebenfalls im Rahmen deutschsprachiger Forschungen verankert, lassen sich diese Einwände und die Ablehnung auch für internationale gewaltsoziologische Forschungen konstatieren (siehe u.a. Collins 2011; Katz 1988, 2020; Fujii 2009, 2013; Ray 2018; Schinkel 2010; Wieviorka 2003, 2014).

4 Vgl. u. a. Armbruster (2016); Braun (2016); Hoebel (2014); Hoebel und Hartmann (2020); Knöbl (2019); Hoebel und Koloma Beck (2019); Koepp und Schattka (2020); Leuschner (2016); Nassauer (2015, 2019); Sutterlüty $(2015,2017)$.

5 Die hier unter dem Begriff des Körperlichen zusammengefassten Ansätze der neueren Gewaltsoziologie zielen im Kern darauf ab, dass individuelle Akteur*innen nicht nur über einen Körper (als Objekt) verfügen bzw. diesen haben, sondern sich diesem (als Subjekt) zugleich selbst-reflexiv bzgl. der (sozial-kulturell überformenden) Umwelt, der (passiven wie aktiven) Handlungen und der Erfahrung wie Wahrnehmung bewusst sind (vgl. u. a. Gugutzer 2004; Koloma Beck 2017; Lindemann 2015; Popitz 1986; Reemtsma 
den Ansätze bereits soziale Kontextfaktoren im Sinne struktureller und kultureller Gewalt berücksichtigen und darüber hinaus - im Gegensatz zu Galtungs (1975a, 1990, 1993b) Konzept - Macht und Gewalt analytisch trennscharf behandeln. Eine solche forschungspraktische Differenzierung ist abgesehen von der bereits erwähnten drohenden tautologischen Gewalterklärung (vgl. Knöbl 2019, S. 35) dabei äußerst sinnvoll. Denn auch wenn die grundsätzliche Verschränkung von Handlungen und Strukturen keineswegs in Abrede gestellt wird (vgl. u. a. Schimank 2000), handelt es sich doch mindestens in

1. zeitlicher (mittelbar [Gewalt]lunmittelbar [Macht]);

2. räumlicher (lokal begrenzt [Gewalt]lglobal/territorial ausgeweitet [Macht]);

3. personaler (explizit Personen gebunden [Gewalt]lüberpersonale Gefüge [Macht]) und

4. prognostischer Hinsicht (logisch-kausale nicht Vorhersagbarkeit des (Nicht-)Handelns [Gewalt] I vorausgesetzte logisch-kausale Annahme der Vorhersagbarkeit des (Nicht-)Handelns [Macht]), um zwei unterschiedliche beobachter*innenabhängige Phänomene. ${ }^{6}$

Ausgehend von einer einführenden Darstellung der galtungschen Gewalttheorie wird der Frage nach dem analytischen Mehrwert struktureller Gewalt für neuere gewaltsoziologische Ansätze in drei Schritten - maßgeblich an den von Imbusch (2017a) vorgetragenen Punkten orientiert - nachgegangen. Basierend auf einem einfachen Vergleich bzgl. des gemeinsamen Bezugsproblems Gewalt wird zunächst auf das Grundproblem der terminologischen Unschärfe Bezug genommen und gezeigt, dass strukturelle Gewalt nicht wie bei neueren gewaltsoziologischen Ansätzen auf violentia rekurriert, sondern als potestas dem Aspekt der Machtausübung zuzuordnen ist. Anschließend wird im zweiten Schritt der von Imbusch (2017a, S. 38) vorgetragene (scheinbar) nicht zu rechtfertigende Körperbezug neuerer Gewaltforschungen betrachtet. Hier wird aufgezeigt, dass selbst in Galtungs (1969, 1975a, 1996) Ansatz sowie mit Blick auf eine potenzielle Erweiterung neuerer Gewaltforschung durch das Konzept der strukturellen Gewalt der Körper weiterhin den zentralen Kern von Gewaltforschungen bildet. Im Kontext körperlicher Gewaltansätze führt dies gegenüber Galtungs (1975a) Konzept dazu, dass strukturelle und (de-)legitimierende kulturelle Kontextfaktoren ohne das normative Konzept der strukturellen Gewalt und ohne Sichtweise auf Gewalt als „,moralisches oder politisches Problem“

2009). Diese Grundauffassung entspricht dabei auch dem von Galtung (u.a. 1985, 1996) betonten (buddhistischen) Verständnis körperlich-mentaler Lebewesen.

6 Vgl. Arendt (1970); Collins (1975, 2011); Luhmann (1992, S. 68-121, 2012, S. 13-39); Popitz (1986, S. 37-106); Weber (1980, S. 28, 122-142, 603-612). Und selbst wenn Gewalt auch als Zweck und/oder Machtmittel - im Zuge der Bildung und Aufrechterhaltung spezifisch legitimierter sozialer Ordnungen verstanden werden kann, so ist deren diesbezüglich tatsächliche Anwendung zum einen doch ,in aller Regel die Ultima Ratio“ (Knöbl 2019, S. 33) und kontextuell (normativ) legitimiert, was die Frage nach nicht justiziablen Gewaltakten in gewisser Weise ausblendet (Vgl. Arendt 1970; Popitz 1986, S. 37-106; Simmel 1992, S. 284-382; Weber 1980, S. 28, 122-142, 603-612). Und zum anderen zeigen Ansätze einer praktisch orientierten Gewaltforschung, die ebenso den ,,präventiven, präemptiven und interventionalistischen Umgang mit Gewalt adressiert“ (Kron 2019, S. 1), dass Gewalt als komplexes Phänomen auch ohne eine analytische Gleichsetzung mit Macht adäquat erklärt werden kann (vgl. u. a. Kron 2019; Staller und Körner 2020; siehe auch Collins 2019). 
(Hartmann 2017, S. 1) berücksichtigt werden können. Drittens wird schließlich auf die Verbindung von struktureller und kultureller Gewalt eingegangen. Hier zeigt sich zum einen, dass gerade die dyadische Täter*innen-Opfer-Konstellation Galtungs (1975a, 1990, 1993b) die Frage aufwirft, wer die erforderliche Differenz von Aktuellem und Potenziellem zur Erklärung von Gewalt beobachtet und (de-)legitimiert. Zum anderen wird verdeutlicht, dass ein dementsprechend fehlendes Beobachter*innenkonzept bei Galtung (1975a, 1990, 1993b) auch durch den Versuch der Einbindung struktureller Dritter (vgl. Imbusch 2017a) die Abgrenzungsproblematik von Macht und Gewalt analytisch nicht trennscharf behandeln lässt, da gerade die zirkuläre Verwobenheit von struktureller und kultureller Gewalt dieses Problem verschärft.

\section{Strukturelle Gewalt - Zur Konzeptualisierung der galtungschen Gewalttheorie}

Zentral für Galtungs (1969, 1975a, 1975b, S. 11-12) einst revolutionäres Konzept der strukturellen Gewalt (vgl. Schmidt 2001) ist ein umfassendes Verständnis und ein universaler, der Multiplizität von Gewalt Rechnung tragender Gewaltbegriff, der die ,theoretisch signifikanten Dimensionen von Gewalt [aufzeigt und; AB] [...] die wichtigsten Varianten einschließt, gleichzeitig aber so spezifisch [ist], daß er die Basis für konkretes Handeln abgeben kann“ (Galtung 1975a, S. 8). Hiervon ausgehend verknüpft Galtung (1975a, S. 8) ,„Frieden“ als ,Abwesenheit von Gewalt‘ [...] und [...] definiert eine friedliche Gesellschaftsordnung nicht punktuell, sondern regional: als den weiten Bereich von Gesellschaftsordnungen, in denen es keine Gewalt gibt" (Galtung 1975a, S. 8). Dabei bezieht sich sein Friedensbegriff auf negativen (direkte physische Gewaltabsenz) und positiven Frieden (Symbiose und Gerechtigkeit in menschlichen Beziehungen im Sinne struktureller und kultureller Gewaltabsenz) (vgl. Galtung 1975a, S. 32-59). Gegenüber den bis dato vorherrschenden Ansätzen verbindet und fordert Galtung (1975a, S. 37-58, 1978a, 1983, 1996) hiermit zugleich einen unverzichtbar logisch-kausal empirisch angeleiteten, theoretische Aussagesätze generierenden und überprüfenden mehrdimensionalen Blick ein, der gleichermaßen in eine normative Sozial- und Gegenwartskritik politisch-sozialer wie kultureller Strukturen mündet (vgl. Brunner 2020, S. 152-162; Jaberg 2019, S. 24-28; Schmidt 2001, S. 507-512): Diese konstruieren auf Basis ihrer (primär westlichwissenschaftlich epistemisch erzeugten) sozio-kulturellen Codes (als zivilisatorische Kosmologien) spezifische Wirklichkeiten, welche in diesem Rahmen und im Sinne von Ungerechtigkeiten, Ungleichheiten, Repressionen und Ausbeutungen letztlich die Selbstentfaltung von Individuen vermeidbar (be-)herrschend konterkarieren. ${ }^{7}$

Auf Basis einer verallgemeinert-marxistischen wie kritisch-theoretischen Fundierung, der analytischen Orientierung an den parsonsschen Strukturvariablen sowie im Zuge seiner Imperialismus- und Kolonialismuskritik verankert Galtung (1971, 1975a, 1978a, S. 15, 289-311, 370, Fn. 17, 1996) jene diversen Formen ,sozia-

\footnotetext{
7 Vgl. Brunner (2020, S. 152-162); Galtung (1978a, 1983, 1996, S. 2, 212-216); Jaberg (2019, S. 24-28); Schmidt (2001, S. 507-512).
} 
le[r] Ungerechtigkeit“ (Galtung 1975a, S. 13) im Kontext weltgesellschaftlicher Zentrum-Peripherie-Relationen und entwickelt zu deren Untersuchung letztlich eine methodologisch relational triangulierende Perspektive. Mit diesem empiristischkritizistisch-konstruktivistischen (Daten-Werte-Theorie) Blick setzt er somit die Wirkung gesellschaftlicher Strukturen - über Aspekte des Imperialismus, Kapitalismus, der Ungleichverteilung von Ressourcen oder ethnischen und geschlechterbezogenen Differenzierungen als Ursachen - mit dem klassischen Verständnis von (physischer) Gewalt gleich. ${ }^{8}$ Diese entdifferenzierende Sichtweise auf Strukturen und Handlungen als Gewalt ermöglicht es schließlich, weltgesellschaftliche Ungerechtigkeiten diagnostisch (datenbasiert) und prognostisch (theoriebasiert) aufzudecken und interventive therapeutische (werte- und theoriebasierte) Handlungsempfehlungen zur Heilung (Reduzierung von Gewalt) und/oder Prävention (Vermeidung von Gewalt) anzugeben (vgl. Galtung 1969, 1975a, S. 7-58, 1978a, 1996, S. 2, 24-29).

Vor diesem (theoretisch-methodologischen) Hintergrund bezieht sich die aus der sozialen Struktur selbst resultierende und ihr innewohnende indirekte sowie ohne sichtbare Akteur*innen auskommende latent wirkende strukturelle Gewalt, welche sich auf intra- und inter-individueller sowie auf intra- und inter-gesellschaftlicher Ebene zeigt, grundsätzlich auf jegliche erfahrungs- und (er-)leidensfähige Lebewesen und ihre natürlichen-materiellen und sozialen Bedürfnisse. ${ }^{9}$ Diese vor allem durch Galtungs (1985, 1993a, 1996) Hinwendung zum Buddhismus entstandene und dessen Potenzial für friedliche Gesellschaftsstrukturen in den Blick nehmende (vgl. hierzu auch Der-Ian Yeh 2006; Senghaas 1998, S. 91-109) Sichtweise ist es schließlich, die ihn (strukturelle) Gewalt - im Gegensatz zur direkten, an sichtbare Akteur*innen gebundene und manifest wirkende personale Gewalt - universell als grundlegend vermeidbare Differenz eines aktuellen und eines potenziellen Zustandes fassen lässt (vgl. Galtung 1969, S. 168-174, 1975a, S. 9-17, 1990, S. 292, 1993b, S. 53-54). Dabei werden entsprechend der methodologischen wie diagnostisch-prognostisch-therapeutischen Trias auch strukturelle und personale Gewalt um einen dritten Aspekt ergänzt bzw. durch diesen gerahmt - die kulturelle Gewalt (vgl. Galtung 1990, 1993b). Hierunter fasst Galtung (1993b, S. 52) all ,,jene Aspekte von Kultur, die dazu benutzt werden können, direkte oder strukturelle Gewalt zu rechtfertigen oder zu legitimieren“ (vgl. Galtung 1990, S. 291). Verstanden als die menschliche Existenz umfassende Sphäre gehören hierzu z. B. Zeichen, Symbole oder auch die Formen westlicher (bzw. global-nördlicher) Sprache, Religion, Ideologie, Kunst und (empirischer/formaler) Wissenschaft (vgl. Galtung 1990, S. 296-301, 1993b, S. 58-69, 1996, S. 196, 201-206). Diese vielfältigen Bereiche kultureller Gewalt fungieren dabei - im Sinne eines „Nährboden[s]“ (Galtung 1993b, S. 58) als Legitimationsbasis für die beiden anderen Gewaltarten und sorgen (als Kosmologien) darüber hinaus dafür, dass jene hiermit verbundenen, ebenso gewalthaften

\footnotetext{
8 Vgl. Brunner (2019, S. 154-158); Galtung (1971, 1975a, 1978a, 1996); Imbusch (2017a, S. 29-30, 37-42); Jaberg (2019, S. 20-21). Im Zuge des galtungschen (1975a, S. 38) Mehrebenenansatzes ist hierfür auch „,der Gedanke des Isomorphismus, d.h. die Annahme, daß, was für eine Ebene gilt, auch für eine andere Ebene gelten könne [Hervorh. im Orig.]“, maßgeblich.

9 Vgl. Galtung (1969, S. 170-171, 1975a, S. 12-13, 17-20, 33, 1975b, S. 11-12, 1990, S. 292, 1993b, S. 53-54, 1996, S. 2, 37-39, 127-195).
} 
Wirkungen (z.B. auch die der gesamten eigenen Kultur) gekonnt verschleiert, institutionalisiert, akzeptabel und moralisch positiv konnotiert werden (vgl. Galtung 1990, S. 296-301, 1993b, S. 58-69, 1996, S. 196, 201-206).

In dieser Gesamtschau konzeptualisiert Galtung (1993b, S. 57) somit ein „teuflische[s] Gewaltdreieck[.]“, in welchem der logisch kausal ableitbare prozessuale Zusammenhang von kultureller (als Invariante) zu struktureller (als Prozess) zu personaler Gewalt (als Ereignis) sichtbar wird (vgl. Galtung 1990, S. 294-295, 1993b, S. 57-58, 1996, S. 15, 199-200). Damit legt er gleichzeitig auch ein zur empirisch angeleiteten Hypothesengenerierung geeignetes Schichtenmodell der Gewalt vor, das der Komplexität von Gewalt bzgl. der expliziten kulturell-strukturellen Kontextualisierung rein physisch-personaler (direkter) Gewalt - und somit negativem und positivem Frieden - (universal) Rechnung trägt (vgl. Brunner 2020, S. 152-181; Galtung 1990, S. 294-295, 1993b, S. 57-58, 1996, S. 15, 199-200).

\section{Zum analytischen Mehrwert struktureller Gewalt im Kontext der neueren Gewaltsoziologie}

Ohne Frage konzeptualisiert Galtung (1969, 1975a, 1985, 1993a, 1993b, 1996) eine mehrdimensionale und zugleich universale Theorie, die Gewalt - und hier gerade auch deren nicht justiziable Formen und Facetten - im Kontext weltgesellschaftlicher Zentrum-Peripherie-Relationen in den Blick nimmt und mithilfe der entdifferenzierenden Sichtweise zugleich die Frage nach der Herstellung von Frieden (politisch) interventiv, nicht nur mit der reinen Abwesenheit von Gewalt, sondern mit dem Aufbau und der Existenz (welt-)einheitlicher stabiler harmonischer Strukturen verbindet.

Maßgeblich geprägt durch den spezifisch historisch-epistemischen Entstehungszusammenhang der galtungschen Theorie, der sich durchaus von neueren Ansätzen der soziologischen Gewaltforschung unterscheidet ${ }^{10}$, stellt sich bzgl. der Frage nach dem analytischen Mehrwert einer konzeptuellen Reintegration somit aber auch grundsätzlich das Problem der Vergleichbarkeit. Schließlich kann der analytische Mehrwert nur dann entdeckt und geprüft werden, wenn unterschiedliche Theorien oder theoretische Standpunkte über ein angebbares Vergleichskriterium miteinander in Beziehung gesetzt werden können (vgl. Anicker 2017, S. 73). Mit anderen Worten geht es also vor dem Hintergrund der Frage nach einem gemeinsam geteilten Bezugsproblem um „das Herstellen von Relationswissen“ (Gresshoff 1999, S. 16;

\footnotetext{
${ }^{10}$ So lassen sich in Ansätzen der neueren Gewaltsoziologie z.B. kaum bis keine theoretischen Anknüpfungspunkte an den Marxismus geschweige denn an Parsons Strukturvariablen finden, eine strikt (linear) logisch-kausale Abfolge oder Zusammenhänge von Gewalt, Strukturen und Kulturen werden nicht geteilt und auch eine dezidiert imperialistisch-kolonialistische Kritik ist in aktuellen Ansätzen nicht integriert (vgl. u. a. Braun 2020; Hauffe und Hoebel 2017; Hoebel und Knöbl 2019; Hoebel und Malthaner 2019). Letzteres kann dabei auch darauf zurückgeführt werden, dass die neuere soziologische Gewaltforschung Gewalt eben nicht als „moralisches oder politisches Problem“ (Hartmann 2017, S. 1) auffasst, was wiederum - und hier liegt durchaus ein gemeinsamer Ausgangspunkt mit Galtungs Arbeiten vor - mit ihrem gesellschaftskritischen Impetus begründet werden kann, der sich gerade bewusst gegen das Narrativ einer „gewaltfreien“ Moderne wendet (vgl. Koloma Beck 2019).
} 
vgl. Abbott 2004; Anicker 2017, S. 73). Wenn also - Imbuschs (2017a) Vorschlag folgend - strukturelle Gewalt für die neuere soziologische Gewaltforschung gewinnbringend sein soll, so ist dies entsprechend nur möglich, wenn eine einheitliche Basis zur Integration bzw. Vergleichbarkeit gefunden werden kann und gewährleistet ist, da ,,ansonsten Missverständnisse, Scheinkontroversen oder ein unsinniges gegeneinander Ausspielen von verschiedenen Positionen vorprogrammiert [sind]" (Greshoff et al. 2007, S. 9).

Bei der hier (kritisch) zu verfolgenden Frage nach dem analytischen Mehrwert der Reintegration struktureller Gewalt in neuere gewaltsoziologische Ansätze fungieren somit - in Anlehnung an eine auf die Entdeckung ausgerichtete theoretisierende Perspektive - der aus der jeweiligen Problembeobachtung heraus resultierende gewählte Gegenstand (als basaler Ausgangspunkt der Forschung) und damit verbunden die diesen benennenden (definierende) Begrifflichkeit als anleitende Vergleichskriterien (vgl. Anicker 2017, S. 73; Swedberg 2012, 2014, S. 16, 2016). Im Sinne Fabian Anickers (2017, S. 73) handelt es sich hierbei um einen ,einfache[n] Vergleich“, bei dem die ,Vermittlung der Bezugsprobleme in einem übergreifenden Problemkontext" (Anicker 2017, S. 76) in den Blick genommen wird. Dieses Vorgehen erweist sich bzgl. der Frage nach dem analytischen Mehrwert insofern als fruchtbar, als zum einen die Problematiken (empiriegelagerter) falsifikationistischer und (theoriegelagerter) hermeneutischer Vergleiche umgangen werden können (vgl. Anicker 2017, S. 73-77). Zum anderen lassen sich hiermit aber auch die im Rechtfertigungskontext der Forschung vorliegende Behandlung theoretischer Konzepte als grundlegend divergent vermeiden und - fasst man diese zudem im Anschluss an Andrew Abbott (2004, S. 162) generell als Heuristiken mit einer ,fraktalen Natur ${ }^{611}$ auf - schwer zu lösende wissenschafts- oder metatheoretische Vergleiche umgehen, sodass zugleich das Potenzial der von Imbusch (2017a) vorgeschlagenen Reintegration eben im Entdeckungskontext in den Blick genommen werden kann (vgl. Abbott 2004, S. 162-210; Anicker 2017; Swedberg 2012, 2014, 2016).

Sowohl in der Sichtung des Konzepts struktureller Gewalt als auch der neueren gewaltsoziologischen Ansätze lässt sich das komplexe Phänomen Gewalt als übergreifender Problemkontext beobachten, wobei sich das Bezugsproblem (als forschungsanleitendes Puzzle; vgl. Abbott 2004) bei beiden (zunächst) auf den bloßen, direkt gewalthaften Akt als Ereignis bezieht, dieses aber mit Blick auf Lösungsstrategien zugleich hinsichtlich der „Umweltbedingungen mit Handlungsrelevanz“ (Tranow 2018, S. 24) kontextualisiert wird. ${ }^{12}$ Auch wenn dies bzgl. Galtung (1969, 1975a, 1996) zunächst irritierend erscheinen mag, liegt sein Fokus doch auf der Frage nach der Herstellung von Frieden im Kontext weltgesellschaftlich existen-

\footnotetext{
11 Abbott (2004, S. 162-210) zufolge lassen sich unterschiedliche Methoden, Methodologien, Paradigmen und damit verbundene Erklärungsprogramme generell als Heuristiken verstehen, da letztlich alle ,central debates of social science are themselves widely (if implicitly) used in a heuristic mode to open new questions and possibilities“ (Abbott 2004, S. 167). Diese Heuristiken weisen dabei zugleich eine fraktale Natur auf, da sich bestimmte Aspekte, die nach außen im Rechtfertigungskontext vermeintlich kritisiert und/oder abgelehnt werden, dennoch bei internen Diskussionen wiederfinden lassen (Abbott 2004, S. 162-210).

12 Vgl. Braun (2020); Galtung (1969, 1975a, 1990, 1993); Hoebel und Knöbl (2019); Koloma Beck (2011, 2015, 2017); Kron (2019); Lindemann (2014, 2015, 2017); Reemtsma (2009); Sutterlüty (2015, 2017); Tranow (2018).
} 
ter globaler Ungerechtigkeits- und Herrschaftsverhältnisse, so ist doch auch sein konzeptueller Ausgangspunkt unmittelbar mit direkter physischer Gewalt verbunden (vgl. Brunner 2020, S. 156ff.; Galtung 1969, S. 167f., 1975a, S. 7ff., 1996, S. 1-2, 32). Denn das Leiden und die Verletzbarkeit von Lebewesen sowie die Definition von (negativem/positivem) Frieden rekurrieren - verbunden über die diabolische Gewalttrias - auf sichtbare, direkte, intendierte physische Gewalt im Kontext interaktionaler Beziehungen, die erst dann logisch kausal mit überpersonalen indirekten und nicht-intendierten strukturellen (als Übertragungsmedium für Gewalt) sowie kulturellen Aspekten (als Quellen der Gewaltlegitimation) erweitert werden (vgl. Brunner 2020, S. 152-181; Galtung 1969, S. 167-174, 1975a, S. 9-17, 1993b, S. 57-58, 1996, S. 2, 8-9).

Vor diesem Hintergrund ist also danach zu fragen, ob strukturelle Gewalt auf Gewalt im Sinne stürmischer und auf Verletzung abzielender Gewaltsamkeiten gemäß des Terminus vis/violentia fokussiert, wie dies bei neueren Ansätzen der Gewaltforschung der Fall ist ${ }^{13}$, oder auf der Ebene der via ungleich verteilter Mittel beruhender (delegierten) Verfügungsgewalt, gemäß des Terminus potestas im Sinne von Machtund Herrschaftsausübung anzusiedeln ist (vgl. Crowther 1995, S. 1268; Imbusch 2000, S. 31-32, 2002, S. 29, 2005, S. 26-27).

\subsection{Das terminologische Grundproblem: Strukturelle Gewalt im Spannungsfeld von potestas und violentia}

Ausgehend vom konzeptuell anleitenden Bezugsproblem kann ein erster Einwand bzgl. der Reintegration struktureller Gewalt in neuere gewaltsoziologische Ansätze auf der damit verbundenen benennenden (etymologisch-semantischen) Ebene verortet werden. Hierzu ist noch einmal Galtungs (1969, S. 168-173, 1975a, S. 9-15, 1990, 1993b) Gewaltverständnis in den Blick zu nehmen: Über die vermeidbare Beeinflussung der aktuellen somatischen und geistigen Verwirklichung menschlicher Lebewesen, verglichen mit ihrer potenziellen Verwirklichung, definiert Galtung (1969, S. 167-169, 1974a, S. 11-12, 1975a, S. 7-9) Gewalt als die Ursache dieser Differenz und rechtfertigt so seine über einen rein direkten physischen und manifesten Gewaltbegriff (personale Gewalt) hinausgehende begriffliche Erweiterung (vgl.

\footnotetext{
13 Obgleich sich auch bei neueren gewaltsoziologischen Ansätzen ein nicht mehr nur rein auf den einzelnen situativ-verorteten, direkten physischen Gewaltakt bezogener Fokus konstatieren lässt, ist die Referenz auf körperlich gebundene (intendierte) Verletzbarkeit und Leiderfahrung gemäß der violentia weiterhin zentraler Bestandteil (siehe u.a. Hoebel und Malthaner 2019; Hoebel und Knöbl 2019; Koloma Beck 2017; Lindemann 2014, 2017; Nassauer 2019; Reemtsma 2009; Sutterlüty 2015, 2017). So verweist bspw. Jan Philipp Reemtsma (2009, S. 104-133) im Kontext seiner zeitlich und sozial differenzierenden Sichtweise auf „unterschiedliche Arten des gewalttätigen Zugriffs auf den Körper“ (Reemtsma 2009, S. 107) explizit darauf, ,dass Gewalt zunächst physische Gewalt [ist], der Übergriff auf den Körper eines anderen ohne dessen Zustimmung“ (Reemtsma 2009, S. 104; vgl. Braun 2019a, S. 68). Auch Teresa Koloma Beck (2011, S. 349-354, 2017, S. 62-73) und Gesa Lindemann (2014, S. 245-277, 2017) verdeutlichen in ihren triadisch angelegten Gewaltbeobachtungen und vor dem Hintergrund struktureller und/oder kultureller Rahmungen die zentrale Rolle körperlicher Gewalterfahrungen (Antun/Erleiden) (vgl. Braun 2019a, S. 68f.; 2020). Und ebenso verweisen Thomas Hoebel und Wolfgang Knöbl (2019) in ihrer prozessual erklärenden Perspektive auf die Relevanz erfahrener Verletzbarkeit - sowohl bei und durch anwesende/n wie auch abwesende/n Dritte/n.
} 
auch Imbusch 2017a, S. 30). Gerade weil menschliches Leid ebenso über (indirekte) sozialstrukturelle Parameter - nämlich in Form sozialer Isolation, Armut, Repression etc. - hervorgerufen und aufrechterhalten werden kann, muss dieser Multiplizität mit der Erweiterung um die Ebene der strukturellen (und kulturellen) Gewalt Rechnung getragen werden (vgl. Galtung 1969, 1975a, 1975b, S. 11-12, 1978b, S. 1-2, 1990, S. 291-305; Imbusch 2017a, S. 36). Im Gegensatz zur direkten personalen Gewalt ist strukturelle Gewalt dabei systemimmanent, indirekt, bedarf keiner sichtbaren Akteur*innen, die direkten Schaden ausüben und wirkt aufgrund der diesbezüglich nicht vorhandenen ,klare[n] Subjekt-Objekt-Beziehung“ (Galtung 1975a, S. 13) latent (vgl. Galtung 1969, S. 170-171, 1975a, S. 12-13, 1975b, S. 11-12). Tritt bei struktureller Gewalt somit ,niemand in Erscheinung, der einem anderen direkt Schaden zufügen könnte [...] äußerst sich [die Gewalt; AB] in ungleichen Machtverhältnissen und folglich in ungleichen Lebenschancen [meine Hervorh.]“ (Galtung 1975a, S. 12). Wobei Galtung (1975a, S. 12-13, 19) hier konkret auf die gesellschaftlich ungleich verteilte ,Entscheidungsgewalt bezüglich der Ressourcen [Hervorh. im Orig.]“ (Galtung 1975a, S. 12) abzielt. Damit findet aber eine Gleichsetzung mit Macht statt und zugleich bezeichnet Galtung (1975a, S. 13), „um das Wort Gewalt nicht zu sehr zu strapazieren, die Bedingung der strukturellen Gewalt [...] als soziale Ungerechtigkeit". ${ }^{14}$

An dieser Stelle offenbart sich somit das Grundproblem für eine gewaltsoziologische Reintegration, das ausgehend vom Bezugsproblem personaler Gewalt letztlich in einer terminologisch ,grundsätzlichen Unbestimmbarkeit“ (Imbusch 2017a, S. 35) besteht und damit ,einer theoretischen Unschärfe Vorschub [leistet]“ (Imbusch 2017a, S. 35), die maßgeblich auf die synonyme Behandlung zweier konträrer Begrifflichkeiten zurückgeführt werden kann - nämlich Macht und Gewalt. Schließlich wird bei Galtung

der präzise Gebrauch des Begriffs Gewalt durch teilweise überlappende Konnotationen mit einer Reihe weiterer Begriffe, die sich in einem ähnlichen semantischen Begriffsfeld bewegen (etwa Zwang, Aggression, Konflikt, Macht etc.), aber eben nicht mit Gewalt identisch sind, erschwert [...] [und; AB] eingeschränkt. (Imbusch 2002, S. 27)

Damit bewegt sich strukturelle Gewalt hinsichtlich des konzeptuell anleitenden Bezugsproblems letztlich etymologisch undifferenziert im semantischen Spannungsfeld von potestas und violentia. Es wird also gleichzeitig zwischen den Aspekten einer auf Macht und Machtmitteln beruhenden (delegierten) Verfügungsgewalt (potestas) und einer ungestümen, wilden Gewaltsamkeit (violentia) verortet, wobei gerade Letztere - vor allem im angloamerikanischen Sprachkontext - mit einer intendierten

\footnotetext{
14 Zwar mit einem anderen Fokus als bei Galtung, zeigt sich dies ebenfalls mit einem erweiterten Begriff und Verständnis von Gewalt operierend auch bei Bourdieus (1997, 2005) Konzept der zuweilen als sanfte oder symbolische Gewalt benannten Wirkweise hegemonial-normativer (vor allem männlich geprägter) Herrschaftsstrukturen. Mit Blick auf dessen Relevanz für gewaltsoziologische Forschungen hat Frithjof Nungesser (2017, S. 7) bereits konstruktiv-kritisch gezeigt, dass die ,unklare Verhältnisbestimmung von symbolischen und physischen Gewaltaspekten“ (Nungesser 2017, S. 7) sowie „die begrifflichen Widersprüchlichkeiten keinen rhetorischen Kunstgriff darstellen“ (Nungesser 2017, S. 7), sondern im Sinne eines „pleonatische[n] Oxymoron[s]“ (Nungesser 2017) wenig analytischen Mehrwert aufweisen.
} 
Verletzung einhergeht (vgl. Crowther 1995, S. 1268; Imbusch 2000, S. 31-32, 2002, S. 29, 2005, S. 26-27). ${ }^{15}$

Zwar mag sich eine klare und präzise Abgrenzung von Macht und Gewalt aufgrund des „soziologisch amorph[en; AB]“ (Weber 1980, S. 28) Charakters des Machtbegriffs als potenziell schwierig erweisen, kann aber - wie einleitend erwähnt - unter Berücksichtigung einer zeitlichen, räumlichen, personalen und prognostischen Komponente vorgenommen und damit auf Galtungs (1975a, 1990, 1993b) Konzept übertragen werden. So bedeutet z. B. Macht nach Max Weber (1980, S. 28) ,jede Chance, innerhalb einer sozialen Beziehung den eigenen Willen auch gegen Widerstreben durchzusetzen, gleichviel worauf diese Chance beruht“". Diese Form der Machtausübung ist dabei als sporadisch anzusehen, da zunächst von keiner Wiederholung ausgegangen werden kann und Weber (1980, S. 28) hier nur einzelne Situationen, aber keinen übergreifenden strukturellen Kontext im Blick hat (vgl. Popitz 1986, S. 42). Dieser Machtbegriff rekurriert demnach auf eine personengebundene, zeitlich direkte und damit (auch räumlich) unmittelbare Handlung, was Galtungs (1993b, S. 57) Begriff der personalen Gewalt entspricht. Da sich Weber (1980, S. 28) hierbei zugleich auf ,alle denkbaren Qualitäten eines Menschen und alle denkbaren Konstellationen [bezieht, die; AB] jemand in die Lage versetzen können, seinen Willen in einer gegebenen Situation durchzusetzen“, ist die hiermit verbundene Handlungsausübung auch keineswegs logisch-kausal vorhersagbar. ${ }^{16}$ Demgegenüber zeigt sich die strukturelle (dauerhafte) Kontextgebundenheit von Macht nach Weber (1980, S. 28, 122-142) erst in ihrer präziseren Form der - über Befehle logisch-kausal prognostizierbaren - Herrschaft, die im Anschluss an Heinrich Popitz (1986, S. 42-67) aus einer prozessualen Entwicklung der sporadischen Macht über wiederholt anwendbare Machtmittel in Kombination mit Leistungsdurchsetzungen hin zu tatsächlich institutionalisierten überpersonalen Erwartungsstrukturen in hierarchischen Positionsgefügen mit unterschiedlichen Lebensbedingungen resultiert. Hier zeigt sich somit eine zeitlich (wie räumlich) mittelbare und demnach latente Wirkung von Macht ohne ,klare[.] Subjekt-Objekt-Beziehung“ (Galtung 1975a, S. 13), was mit Galtungs (1993b, S. 57) Konzept der strukturellen Gewalt identisch ist.

The concept of ,structural violence“ is generally defined as a structure whereby values are generated and distributed asymmetrically so that some kind of verticality arises due to the operation of the structure (as opposed to the deliberate actions of actors within the structure). (Galtung 1974b, S. 23)

\footnotetext{
15 Aufgrund der im deutschsprachigen Kontext vorhandenen undifferenzierten semantischen Verwendung von Gewalt und Macht (vgl. Imbusch 2000, S. 31 f., 2002, S. 29, 2005, S. 26f.) könnte zwar auch von einem Übersetzungsproblem bzw. einem Übersetzungsfehler bzgl. der galtungschen (1975a, 1990) Arbeiten ausgegangen werden. Dies liegt aber nicht vor, da Galtung $(1969,1996)$ selbst die im angloamerikanischen Raum semantisch klar unterschiedenen Begriffe violence (im Sinne von violentia) und power (im Sinne von potestas) (vgl. Crowther 1995, S. 1268) synonym verwendet bzw. in seinen späteren Arbeiten power explizit als ,concept broader than violence, and also broader than peace“ (Galtung 1996, S. 2) einführt.

16 Siehe zur Problematik nicht logisch-kausal vorhersagbarer Handlungen in solch sporadischen Machtsituationen auch Luhmann (2012, S. 17-20).
} 
Jene strukturell bedingte asymmetrische (und damit vertikal hierarchische) Verteilung, die durch die Strukturen selbst (re-)produziert wird, führt über die damit einhergehenden unterschiedlichen Positionierungen in den strukturellen Verteilungsmustern zu unterschiedlichen Lebensbedingungen und somit letztlich zu struktureller Gewalt (vgl. Galtung 1974a, S. 12).

Vor dem Hintergrund der vorherigen Ausführungen ist strukturelle Gewalt demnach aber nicht als Gewalt (im Sinne von violentia), sondern als Macht (im Sinne von potestas) zu verstehen. Dies wird durch den von Galtung (1975a, S. 13) angeführten dritten Begriff der sozialen Ungerechtigkeit als Bedingung für strukturelle Gewalt gestützt: Als nicht moralisch konnotierter und auf Ressourcen (z. B. Einkommen, Bildung) bezogener Begriff im Kontext einer diesbezüglich ungleich verteilten Entscheidungsgewalt verweist Galtung (1975a, S. 12-13) hiermit letztlich synonym auf den Aspekt sozialer Ungleichheit. Schließlich beruhen auch soziale Ungleichheiten auf differenzierten gesellschaftlichen Positionengefügen und einer damit verbundenen differenten (demnach ungleichen sowie ungerechten) Güterverteilung auf Basis überpersönlicher Mechanismen (vgl. Hradil 2016, S. 248-249). Auch dies entspricht somit den obigen Ausführungen zu Macht. Neben der auf der Benennung des Bezugsproblems beruhenden terminologischen Schwäche wird damit zugleich das von Imbusch (2017a, S. 35) konstatierte Problem der widersprüchlichen Definition von struktureller Gewalt als Wirkung und Ursache deutlich. In analytischer Hinsicht besteht hier somit kein erkennbarer Vorteil: Statt Bestrebungen einer Präzisierung des Forschungsgegenstandes - im Sinne der trennscharfen Behandlung von Gewalt als eigenständigem Phänomen (vgl. Arendt 1970, S. 36) - bleibt die gewaltsoziologisch einst kritisierte nicht bestimmbare Ausdehnung auf alle denkbaren Formen von Gewalt, Macht und Herrschaft im Spiegel globaler Ungleichheiten bestehen (vgl. Knöbl 2019, S. 32-35). So wären Kredite, die im Kontext befristeter Arbeitsverhältnisse nicht bewilligt werden, die Entwicklung und Existenz von Prüfungsordnungen für Studiengänge oder die gesundheitsschädigende Belastung durch Feinstaub ebenso als Gewalt einzustufen wie Enthauptungen durch terroristische Gruppen oder Überfälle auf offener Straße. Zumindest ist in all diesen Fällen aufgrund der Verortung in differenten hierarchischen Positionengefügen und einer Ungleichverteilung der Entscheidungsgewalt die ,,aktuelle somatische und geistige Verwirklichung geringer als ihre potentielle Verwirklichung" (Galtung 1975a, S. 9).

Trotz des gemeinsamen Bezugsproblems erweist sich die Reintegration des Konzepts struktureller Gewalt in neuere Ansätze der Gewaltforschung somit als wenig vielversprechend, da lediglich der Aspekt personaler Gewalt den Kern einer auf körperlicher Gewalt beruhenden neueren Gewaltsoziologie bzgl. der notwendigen Vergleichbarkeit widerspiegelt. Ein solitär hierauf beruhender Fokus ist aber aufgrund der strikt als Gewalttrias zu denkenden Konzeptualisierung Galtungs (1990, 1993b) ausgeschlossen (vgl. Imbusch 2017a, S. 35).

\subsection{Die Fokussierung auf Körper und Leid als problematische Eingrenzung? Abgrenzungsprobleme struktureller und physischer Gewalt}

Ein zweiter Einwand richtet sich - ebenfalls ausgehend vom Bezugsproblem - auf die von Imbusch (2017a) kritisierte gewaltsoziologische Engführung auf den Körper. 
Zwar weist Imbusch (2017a, S. 35) zu Recht darauf hin, dass strukturelle Gewalt personale Gewalt nicht ersetzen, sondern um eine weitere Dimension nebst der kulturellen Gewalt ergänzen will (vgl. auch Galtung 1969, 1975a, 1990, 1996). Aber gerade die körperliche Komponente, die nicht nur von der neueren Gewaltforschung fokussiert wurde (vgl. u.a. Popitz 1986; Reemtsma 2009; Sofsky 1996, 2002; von Trotha 1997), sondern auch in Galtungs (1969, 1975a, 1996) Konzept als Ausgangspunkt angelegt und ebenso für seine weiteren Überlegungen zentral ist, bietet Anlass zu einer weiteren Hinterfragung des analytischen Gewinns. Denn sowohl bzgl. der personalen und strukturellen als auch der kulturellen Gewalt ${ }^{17}$ ist die somatische und mentale Beeinflussung von Personen relevant (vgl. Galtung 1969, 1975a, S. 35, 1978a, 1990, 1993b, 1996, S. 2, 9).

So führt Galtung (1975a, S. 13) bspw. an: „Wenn also ein Ehemann seine Ehefrau schlägt, dann ist das ein klarer Fall von personaler Gewalt; wenn aber eine Million Ehemänner eine Million Ehefrauen in Unwissenheit halten, dann ist das strukturelle Gewalt“". In beiden Fällen zeigt sich die Ausübung von Gewalt gemäß der Definition in der Unterbindung und Vermeidung des Aktuellen und Potenziellen. Im ersten Fall hinsichtlich der potenziellen körperlichen Unversehrtheit in Paarbeziehungen, die durch das vermeidbar Aktuelle (hier das Schlagen des Ehemannes) unterbunden wird. Im zweiten Fall hinsichtlich der potenziellen mentalen Weiterbildung aufgrund des Zurückhaltens spezifischer Informationen (das Aktuelle), was ebenfalls vermeidbar ist. Im Kontext dieses konzeptuell verankerten expliziten Körperbezugs - zumindest aufseiten der Opfer - stellt sich somit die Frage, ob die von Imbusch (2017a, S. 38) kritisch betrachtete Engführung auf den Körper als Fixpunkt neuerer Gewaltforschungen nicht doch gerechtfertigt ist. Denn während sich lediglich die sozialen Ursachen in den beiden obigen Fällen unterscheiden - individuell-personale Motivlage (sporadische Macht in Form manifester physischer Gewalt) versus institutionalisierte (männliche) Herrschaftsstrukturen - ist der Effekt der scheinbar differenten Aktionen identisch: ein Einwirken auf Individuen in struktureller (Anatomie) und/oder funktionaler (Physiologie) Hinsicht (vgl. Galtung 1975a, S. 18). Diese beiden Dimensionen verortet Galtung (1975a, S. 19-21) in der personalen Gewalt, obwohl - und hier zeigt sich erneut die konzeptionelle Unbestimmtheit - der körperliche Funktionsbezug zugleich in die ordnungsstiftenden Mechanismen struktureller Gewalt eingebunden ist. So zielen z. B. auch strukturell verursachte Hungersnöte, (sozial-)räumliche Exklusionen in Form von Gettoisierungen oder Armenvierteln oder der Abkauf von Brunnen- und/oder Wasserrechten in Teilen Afrikas - alles im galtungschen Sinne Formen struktureller Gewalt - letztlich auf die funktionsbezogenen Dimensionen personaler Gewalt ab; nämlich in Form des Entzugs von Wasser, Nahrung und Bewegung (vgl. Galtung 1975a, S. 19, 1996; Imbusch 2017a, S. 37-38; Schroer 2004, S. 169).

Imbusch (2017a, S. 38) zufolge könne aber gerade in Anlehnung an Markus Schroer (2004) der Blick auf strukturell bedingte (Multi-)Exklusions- und/oder Marginalisierungserfahrungen das Potenzial und ,die Bedeutung eines Begriffs der strukturellen Gewalt für die Gewaltforschung“ (Imbusch 2017a, S. 38) stärken, da dieser

${ }^{17}$ Hier hinsichtlich der (mental) epistemisch erzeugten zivilisatorischen Kosmologien als basale, legitimierende sozio-kulturelle Codes. 
„eine nicht zu rechtfertigende Engführung des Gewaltverständnisses vermeidet und sichtbar macht, dass Gewalt eben nicht in physischer Gewalt aufgeht" (Imbusch 2017a, S. 38).

Wäre aber, wenn schließlich auch strukturelle Gewalt in letzter Instanz auf den Körper einwirkt und somatisch erfahren wird und damit jegliche Form von Elend auf erfahrungs- und leidensfähige Lebewesen rekurriert (vgl. Galtung 1975a, S. 19-21, 1996, S. 2), nicht gerade die explizite Fokussierung auf einen mit situativem Schmerz und Leiderfahrungen verbundenen Begriff physischer (im Sinne körperlicher) Gewalt (weiterhin) analytisch fruchtbarer?

Diese Überlegung wird zumindest von drei Aspekten gestützt: Erstens hat „die Engführung des Gewaltbegriffs, also die Fokussierung auf physische Gewalt [...] tatsächlich zu neuen und originären Einsichten geführt“" (Knöbl 2019, S. 34) und alleine der Rekurs auf zeitliche Bedeutungsverschiebungen rechtfertigt noch keine universale Ausweitung des Gewaltbegriffs auf sämtliche nur denkbaren Formen von Ungleichheiten, Marginalisierungen usw., wenn nicht zugleich aufgezeigt wird, ,was sich an analytischer Präzision gewinnen ließe“ (Knöbl 2019, S. 34; vgl. Knöbl 2019, S. 34-35). Die Wahl eines weiten Gewaltbegriffs aufgrund seiner alleinigen politischen Wirkmächtigkeit repräsentiert schließlich keine analytische Begriffsschärfe (vgl. Brock 1995, S. 344). Dies widerspricht dabei nicht grundsätzlich Imbuschs (2017a) Vorschlag, sozialstrukturelle Parameter oder generelle soziale Kontextfaktoren zur Erklärung von Gewalthandlungen zu berücksichtigen. Eine derart gelagerte Erforschung von Gewalt kann aber unter Rekurs auf diverse ungleichheits- und machttheoretische oder andere sozialstrukturelle Konzeptionen vorgenommen werden, ohne hierbei analytisch mit dem ,stark [...] normativ aufgeladen[en]“ (Imbusch 2017a, S. 36) Konzept Galtungs zu operieren (vgl. Knöbl 2019, S. 33-35).

Zweitens sind Strukturen, obwohl handlungsprägend, selbst nicht handlungsfähig (vgl. u.a. Schimank 1985). Denn als Resultat von aus handlungsfähigen Akteur*innen und deren Konstellationen ([un-]intendiert) gebildeten komplexitäts- und kontingenzreduzierenden Handlungsmaximen, die letztlich stabile, allgemeingültige, normativ verankerte Erwartungen produzieren, benötigen so geschaffene Strukturen für die Umsetzung bzw. Reproduktion ihrer (kulturellen/strukturellen) Rahmungen stellvertretend agierende kollektive oder korporative Akteur*innen (vgl. Berger und Luckmann 2000, S. 49-98; Braun 2015, S. 65-81, 145; Göhler 1997; Lepsius 1995, S. 393ff., 1997, S. 57 f.; Schimank 1985, 1988, 2000, S, 173-322). Schließlich kann die Stabilität von Strukturen ,,nur dann gewährleistet werden, wenn die allgemeine Gültigkeit der Handlungsmaximen durch Akzeptanz, Zustimmung und vor allem Internalisierung zu dauerhaft verfestigten Verhaltensmustern führt, welche eben die regulierende und orientierende Funktion [...] [jener Strukturen; AB] gewährleisten“ (Braun 2015, S. 145). Dies erfolgt über Organisationen und Institutionen und deren Mitglieder bzw. Angehörige, die wiederum aufgrund ihrer Handlungsfähigkeit die strukturellen Rahmungen im Kontext von Interessenkonstellationen und Intentionsinterferenzen erhalten, anpassen, aber auch reflexiv-rekursiv - eben aufgrund der (re)produktiven Verwobenheit von Handlungen und Struktur - verändern können (vgl. Braun 2015, S. 65-81; Geser 1990; Schimank 1985, S. 427-430; Giddens 1984, S. 19, 25; Gukenbiehl 2002; Luhmann 1964; Meyer und Rowan 1977; Münch 1984, S. 7-20, 1991, S. 306f.). Vor diesem Hintergrund wird somit ersichtlich, dass auch 
die Ausübung (indirekter) struktureller Gewalt letztlich durch (direkt handlungsfähige) Stellvertreter*innen erfolgt und dadurch (im doppelten Wortsinn) verkörpert wird - sei es bspw. durch ([in-]direkte) Entscheidungen in organisationalen Kontexten oder als ([in-]direkte) strukturelle Repräsentant*innen (vgl. Braun 2019a, S. 71). So benötigen z. B. (post-)kolonialistisch-imperialistisch motivierte Landvertreibungen, die bewusste Zurückhaltung (oder gar Vernichtung) von spezifischem Wissen oder der kapitalistisch motivierte Abkauf von Wasser- und Brunnenrechten in Afrika ebenso (militant) agierende Stellvertreter*innen vor Ort wie auch (sozial-)räumliche Exklusionen, z. B. in aktueller Form der Gentrifizierung, nicht ohne das (spekulative) Agieren stellvertretender Akteur*innen denkbar sind (vgl. u. a. Bourdieu 2005; Brunner 2020, S. 60-73; Kern 2015; Nixon 2011). Hierauf verweist auch Galtung (1975a, 1990, 1993, 1996, S. 29-34), wenn er trotz des systemimmanenten Charakters:

a) strukturelle Gewalt (im Sinne von Verkörperung) als „,built into the person“ (Galtung 1996, S. 31) definiert;

b) auf die von Akteur*innen produzierte motivierende Kraft kultureller Gewalt rekurriert, die dazu dient, strukturelle Gewalt nicht zu bekämpfen und diese

c) explizit an unterschiedliche (korporative) Trägerschaften sowie deren (handlungsfähige) Mitglieder (z. B. top-dogs) zur Reproduktion struktureller Gewalt gebunden ist - zumal sich die hiermit verbundenen Marginalisierungs- und Ungerechtigkeitserfahrungen im Kontext sozio-kulturell variabler Rahmungen prinzipiell auch jederzeit in physischer Gewalt äußern können.

Drittens geht die Perspektive, welche die körperliche Erfahrung und Ausübung von Gewalt mit sozio-kulturellen Umwelterfahrungen und -kontexten verbindet, bereits (analytisch präzise) mit neueren Ansätzen der Gewaltforschung einher. ${ }^{18}$ Denn gerade hier wird unter physischer Gewalt ein Schmerz und Leid verursachender externer Eingriff auf Personen verstanden, der zugleich an die situationsspezifische subjektive Erfahrung, Deutung und Wahrnehmung dieses Eingriffes als Gewalt und Schmerz im Kontext sozialer Umwelten gebunden ist: eben als körperliche (Gewalt-)Erfahrung. ${ }^{19}$ So verweist bspw. Gesa Lindemann $(2014,2015,2017)$ in ihrer triadisch angelegten Gewaltkonzeption auf die räumliche, sachliche, zeitliche und symbolische Bestimmung der Akteur*innen durch Umweltbezüge und geltende Verfahrensordnungen in der Situation der Gewaltanwendung. Teresa Koloma Beck $(2011,2015,2017)$ verweist ebenfalls auf die Bedeutung sozialer, kultureller und politischer Bedingungen, welche nicht nur für die Beobachtung und damit (Un-)Sichtbarmachung von Gewalt, sondern auch für deren reflektierte Erklärung - im Sinne einer Indexikalität von Gewalt - von Relevanz sind (vgl. auch Hoebel und Koloma Beck 2019). Und auch Thomas Hoebel und Wolfgang Knöbl (2019, S. 173) integrieren in ihrem prozessualen Ansatz ,situierte [...] und kontextgebundene" Handlungsspezifika und nehmen damit konkrete Handlungsorte, externe

18 Vgl. u.a. Braun (2019b); Kersten (2020); Koloma Beck (2011, 2015, 2017); Kron (2020); Lindemann (2014, 2015, 2017).

19 Vgl. Inhetveen (2017, S. 104-111); Lindemann (2014, S. 245-277, 2015, S. 504-506, 2017); Koloma Beck (2017, S. 67); Popitz (1986, S. 68-106); Sofsky (1996). 
Bedingungen, zeitlich-relationale (Beziehungs-)Verstrickungen sowie antizipierende Deutungs-, Bewertungs- und Wahrnehmungsmuster (extra)lokaler Akteur*innen in den Blick, die zudem durch übersituational geltende (generalisierte) „Prämissen des Handelns und Erlebens“ (Hoebel und Knöbl 2019, S. 167) gestützt werden (vgl. Braun 2020; Hoebel und Knöbl 2019: S. 159-175).

Vor diesem Hintergrund scheint Imbuschs (2017a) Plädoyer zur Aufhebung eines expliziten Körperbezugs in der neueren Gewaltsoziologie bzw. zu dessen Erweiterung über den einst abgelehnten Begriff der strukturellen Gewalt nicht zwingend überzeugend. Selbst in den von Imbusch (2017a) angeführten Ausführungen Markus Schroers (2004) liegt der Fokus auf einer leidensbasierten Reduktion auf den Körper (vgl. Imbusch 2017a, S. 37-38; Schroer 2004, S. 166-171). Am Beispiel der multiexkludierten Personen in brasilianischen favelas zeigt sich die „Gewalt, die die Ausgeschlossenen ihrer Persönlichkeitsrechte beraubt und nur noch als bloße Körper behandelt" (Schroer 2004, S. 167) zum einen in ihrer Wirkung hinsichtlich funktionsbezogener - und damit nach Galtung (1975a, S. 18) physiologischer Einschränkungen der Individuen bzgl. täglicher Hungererfahrungen oder räumlicher Mobilitätseinschränkungen (Schroer 2004, S. 166-167). Im galtungschen (1975a, S. 18) Sinne handelt es sich hierbei somit um personale Gewalt(ein)wirkung. Zum anderen erfolgt aber auch die externe Beobachtung und Wahrnehmung der strukturellen Gewalt in Form der Multiexklusion über mediale Berichterstattungen und damit ebenfalls über den Aspekt der körperbezogenen personalen Gewalt (Schroer 2004, S. 166-169).

Der Körper ist oft das einzig verbleibende Kapital, das die [...] Betroffenen im Kampf um die knappe Ressource Aufmerksamkeit einzusetzen vermögen. Womit sonst erreicht man Aufmerksamkeit? Medien reagieren auf einfach darstellbare Sachverhalte. Blutverschmierte Körper, prügelnde Fäuste, schreiende Münder sind nicht zu ignorieren. (Schroer 2004, S. 169)

Findet sich demnach auch bei Schroer (2004) trotz der Berücksichtigung des strukturellen Gewaltkontextes eine Fokussierung auf erleidende Körper, übersehen demgegenüber Ansätze der neueren Gewaltsoziologie keineswegs soziale Kontextund Umweltfaktoren. Im Gegenteil: Trotz oder gerade aufgrund einer Fokussierung auf physische Gewalt und/oder somatische Erfahrungen sind diese in der Lage, soziale Umwelt- und Kontextfaktoren analytisch zu integrieren.

Imbuschs (2017a, S. 38) Kritik, dass „Gewalt eben nicht in physischer Gewalt aufgeht“, wird somit bereits von neueren gewaltsoziologischen Ansätzen berücksichtigt. Zwar ist mit dem Fokus auf körperliche Gewalterfahrungen „Gewalt zunächst physische Gewalt, der Übergriff auf den Körper eines anderen ohne dessen Zustimmung“ (Reemtsma 2009, S. 104), aber jenes ,,,zunächst“ heißt, dass sich auch unsere Vorstellung von nichtphysischer Gewalt an physischer orientiert" (Reemtsma 2009, S. 104). Damit umfasst der Begriff der physischen Gewalt - im Sinne einer breiter gedachten körperlichen Gewalt - ebenso Aspekte psychischer, sexueller oder anders gelagerter bedrohlicher Übergriffe wie z.B. als unangenehm empfundene Blickkontakte, erzwungene räumliche Einschränkungen oder Verbringungen oder soziostrukturell legitimierte sexuelle Übergriffe in Paarbeziehungen (vgl. Koloma Beck 2017, S. 66-67; Lindemann 2017, S. 71-73; Reemtsma 2009, S. 104-124). 
Wohlgemerkt widerspricht dies nicht dem Kern der von Imbusch (2017a) angeführten Sichtbarmachung und Konzentration auf soziale Bedingungen bei der Erklärung von Gewalt. Die grundlegende somatische Basis muss diesbezüglich aber keineswegs aufgegeben werden. Sie rechtfertigt gewissermaßen sogar weiterhin einen starken Bezug auf körperliche Gewalt, der zugleich mit Kontextbedingungen ergänzt werden kann (vgl. Knöbl 2019, S. 35; Koepp und Schattka 2020) - und dies, ohne dabei mit dem theoretisch unscharfen normativen Konzept struktureller Gewalt operieren zu müssen (vgl. Imbusch 2017a, S. 35-36).

Unter Rekurs auf das Bezugsproblem und mit Blick auf die Frage nach dem analytischen Mehrwert ist das Plädoyer für eine Erweiterung bisheriger gewaltsoziologischer Ansätze somit auch hier wenig vielversprechend.

\subsection{Gewaltbeobachtungen zwischen Struktur und (De-)Legitimation - Abgrenzungsprobleme struktureller und kultureller Gewalt}

Bei neueren gewaltsoziologischen Ansätzen beruht die vom Bezugsproblem ausgehende Benennung eines Gewaltereignisses aber nicht nur auf körperlichen Akteur*innen und sozialen Kontextfaktoren. Gewalt ist hier ebenso stets im Kontext einer retrospektiven (prozessualen) (Selbst-)Beobachtung und Einschätzung des Ereignisses durch die Beteiligten selbst als auch durch (selbst-)beobachtende (personale, institutionale und/oder organisationale) Dritte zu sehen. ${ }^{20}$ Diese (re-)konstruieren die (de-)legitimierende Korrelation der zufügenden Aktion mit dem erleidenden Schmerz in Abhängigkeit von beobachter*innenspezifischen sozialen Normen und individuellen Werten. ${ }^{21}$ Im Gegensatz zur normativ aufgeladenen und definiten a priori Täter*innen-Opfer-Zuschreibung Galtungs (1975a) sowie der konzeptuell vorherrschenden Ambivalenz und Unbestimmtheit von Gewalt und Macht sind diese somit in der Lage, genau jene Ambivalenz und Unbestimmtheit auf Basis einer sozialtheoretischen Fundierung zu entwirren, weil sich gerade im nicht von vorneherein fest bestimmten Beobachter*innenmodus der Wechsel von der transitiven Bedeutung der violentia hin zur intransitiven Bedeutung der potestas vollzieht (vgl. Koloma Beck 2011, S. 350-351). Dies schließt nicht nur die prozessuale Dynamik gewalthafter Interaktionen, einen präzisen körperlichen Gewaltbegriff (violentia) und den (symbolisch-)kommunikativen Akt von Gewalt ein, sondern berücksichtigt zugleich die unterschiedlichen (de-)legitimierenden Zuschreibungsmöglichkeiten im Kontext spezifisch hiervon abgrenzbarer (institutionalisierter) Machtstrukturen (potestas). ${ }^{22}$ Die Berücksichtigung (personaler, institutionaler und/oder organisationaler) Dritter als sozial konstitutives Element kann zudem kritisch-(selbst-)reflexiv blinde Flecken in der (Selbst- bzw. Fremd-)Beobachtung aufdecken, jenseits einer rein subjektiv oder objektiv gelagerten Perspektive helfen, vermeintliche Objektivität zu objekti-

\footnotetext{
20 Vgl. Hartmann und Hoebel (2020); Hoebel und Knöbl (2019); Imbusch (2005, S. 136-137, 2017a, 2017b); Koloma Beck (2011, S. 349-351, 2015); Lindemann (2017, 2018, S. 60-82); Nedelmann (1997, S. 72-80); Reemtsma (2009, S. 467-505).

21 Vgl. Imbusch (2005, S. 136-137, 2017b); Koloma Beck (2011, S. 349-351, 2015); Lindemann (2017); Nedelmann (1997, S. 72-80); Reemtsma (2009, S. 467-505).

22 Vgl. Koloma Beck (2011, S. 351); Lindemann (2015, S. 505-506, 2017); Reemtsma (2009, S. 467-505).
} 
vieren und zugleich Bedeutungsverschiebungen von Gewalt im Kontext überpersonal institutionalisierter und zeitlich variierender sozio-kultureller Strukturen analytisch präzise erfassen und sichtbar machen. ${ }^{23}$

Eine derart klare analytische Trennung von Macht und Gewalt sowie die situativkontextuale Berücksichtigung der (De-)Legitimation findet demgegenüber in Galtungs (1975a, 1990, 1993b) dyadischer Täter*innen-Opfer-Konstellation nicht statt, was erneut die Frage nach dem analytischen Mehrwert aufwirft: Bei Galtung (1975a, 1990, 1993b, 1996) bleibt weitestgehend unklar, wer wen wie beobachtet und somit die notwendige Differenz von Aktuellem und Potenziellem für die (de-)legitimierende Zuschreibung von personaler und struktureller Gewalt auf Basis der kulturellen Gewalt identifiziert und vornimmt. ${ }^{24}$ So liegt Galtung (1975a, S. 10, 12) zufolge z.B. bei einer niedrigen Alphabetisierungsrate strukturelle Gewalt vor, da dieser aktuelle Zustand prinzipiell vermeidbar ist. Ob es sich hierbei aber tatsächlich um wahrgenommene und erfahrene Gewalt aufseiten der Betroffenen handelt, ist unklar. Im Gegensatz zur Erfahrung von direktem physischen Schmerz und Leid in Form direkter Einwirkungen auf den Körper (z. B. durch Schläge, Tritte oder Waffen), deren kognitive Wahrnehmung wie Empfindung auf eine Grenzüberschreitung des normalen somatischen Zustands hinweist und somit auf einem subjektiven Wissen hinsichtlich der Divergenz von Aktualität und Potenzialität beruht, ist dies bei struktureller Gewalt nicht zwangsweise gegeben. Galtung (1975a, S. 10) selbst führt diesbezüglich an:

Die Bedeutung des Begriffes ,,potentielle Verwirklichung“ [ist] besonders dann überaus fragwürdig, wenn er nicht mehr allein auf den physischen Bereich des menschlichen Lebens bezogen ist, [...] sondern vielmehr auf den geistigen. Hier müssten wir uns von der Frage leiten lassen, ob der zu realisierende Wert einigermaßen konsensual ist, wenngleich das auf keinen Fall befriedigend ist. (Galtung 1975a, S. 10)

Selbst wenn laut Galtung (1975a, S. 10), ,die Kenntnis des Lesens und Schreibens [...] überall in hohem Ansehen [steht]" und insofern als konsensual angesehen werden kann, als dies über den generellen Zugang zu Bildung normativ in Artikel 26 der Erklärung der Menschenrechte verankert ist (vgl. Vereinte Nationen 1948), setzt die Auffassung und Wahrnehmung niedriger Alphabetisierungsraten als strukturell er-

\footnotetext{
23 Vgl. Berger und Luckmann (2000, S. 49-98); Bourdieu (1993); Imbusch (2005, S. 136-137, 2017b); Koloma Beck (2011); Lindemann (2014, 2015, 2017, 2018, S. 60-82); Luhmann (1992, S. 68-121); Nedelmann (1997, S. 72-80); Reemtsma (2009, S. 470); Simmel (1992, S. 115-159).

24 Im Zuge seiner Friedenstheorie und der Diagnose-Prognose-Therapie-Trias plädiert Galtung (1996, S. 24-39) zwar für ein gerade der Therapie dienliches, (universales) eklektizistisches Beobachter*innenmodell, bei dem die Beobachtung bzgl. der Sensibilisierung für die und Aufdeckung der vielfältigen Formen weltgesellschaftlicher Ungerechtigkeiten und Ungleichheiten eben nicht einzig und allein (wissenschaftlichen und/oder politischen) Eliten überlassen werden soll, sondern in den (international) unterschiedlichen Sichtweisen und Perspektiven von politischen und wissenschaftlichen Eliten, sozialen Bewegungen sowie der Bevölkerung relational zusammenlaufen muss. Wie dies aber theoretisch (und damit auch analytisch) präzise gefasst werden kann, bleibt allerdings offen. Zudem scheint Galtung auch zu übersehen, dass eine solch gewissermaßen ,synoptische“ Beobachtung wiederum die Gefahr weiterer und neuer sozialer Klassifizierungen, Exklusionen und Ungleichheiten birgt, die damit der Schaffung eines positiven Friedens entgegenstehen (vgl. u. a. Bauman 2003; Bauman und Lyon 2013).
} 
fahrene Gewalt aufseiten der Erleidenden Kenntnis über eine existente Schriftsprache sowie über die Existenz jenes Menschenrechts voraus, um die Divergenz von Potenzialität und Aktualität überhaupt erst zu wissen. Ist dieses Wissen nicht vorhanden, liegt (zumindest) in der Selbstbeobachtung der vermeintlichen Opfer keine Gewalt vor. Wird dieses Wissen hingegen von den Täter*innen strukturell bewusst vorenthalten, liegt also nach Galtung strukturelle Gewalt vor, bedeutet dies der Logik des Gewaltdreiecks folgend, dass diese Form der strukturellen Gewalt einer für die Täter*innen und deren konstatierte Taten legitimierenden kulturellen Gewalt unterliegt (vgl. Galtung 1993b, S. 52-59). Diese hebt schließlich ,die Art und Weise hervor, in der direkte und strukturelle Gewalt legitimiert und für die Gesellschaft akzeptabel gemacht wird“ (Galtung 1993b, S. 53), indem , die ,moralische Färbung “ einer Handlung von rot/falsch auf grün/richtig oder zumindest auf gelb/akzeptabel schaltet“ (Galtung 1993b, S. 53; vgl. S. 57-59). Die auf kultureller Gewalt basierende legitimatorische Basis beruht dabei auf unterschiedlichen beobachter*innenunabhängigen (strikt zweiwertigen) Unterscheidungslogiken: ${ }^{25}$ z. B. auf der nicht hinterfragbaren, auf Religion oder Heiligkeit basierenden, Unterscheidung von gläubig/ungläubig bzw. auserwählt/nicht-auserwählt, der ideologischen oder nationalstaatlichen Unterscheidung auf Basis des Abgrenzungsmechanismus wir/andere oder westlich/nichtwestlich, der ,sprachliche[n] (logozentrische[n]) Unterscheidung zwischen Sein und Schein“ (Baecker 2007, S. 37) oder der wissenschaftlichen wahr/nicht-wahr Unterscheidung auf Basis der mathematischen wie philosophischen Aussagenlogik mit dem inhärenten Prinzip des tertium non datur (vgl. Galtung 1993b, S. 61-68). Liegt aufseiten der strukturellen Machtinhaber*innen diesbezüglich somit kein Verstoß gegen den kulturell legitimierten normativen Rahmen vor, bspw. durch die Deutung und Auffassung der Erleidenden als moralisch nicht zählende Personen, handelt es sich sowohl in der Selbst- als auch der Fremdbeobachtung nicht um Gewalt (vgl. Lindemann 2015, S. 507). Denn ,,wenn eine anerkannte soziale Person ,Gewalt‘ ausübt, wird diese zu einem Fall von Nicht-Gewalt, wenn es sich beim ,Gewaltopfer um Wesen handelt, die nicht in den Kreis derjenigen gehören, die moralisch zählen“ (Lindemann 2015, S. 507). Dies trifft in ähnlicher Weise auch auf die von Schroer (2004, S. 166-171) angeführte Auffassung struktureller Gewalt als „,multiplikatorische Exklusion [Hervorh. im Orig.]“ (Schroer 2004, S. 167) zu. Hier können die Erleidenden aufgrund ihrer bewussten (mindestens räumlichen) Marginalisierung seitens der strukturellen Machtinhaber*innen als moralisch nicht zählende Perso-

\footnotetext{
25 Die an dieser Stelle konstatierte beobachter*innenunabhängige Existenz der Unterscheidungslogiken rührt daher, dass sie als langfristig stabile, der kulturellen Gewalt inhärente soziale Codes schwer zu (verändern sind und Galtung (1990, 1993b, 1996) diese (westlich geprägten) Logiken - sowie deren epistemische gewalthafte Wirkweise - zum einen aus seiner Beobachterperspektive als grundsätzlich gesetzt und im weltgesellschaftlichen Kontext als universal gültig sieht. Zum anderen ist dies auch darauf zurückzuführen, dass jene sozialen Codes über den Prozess der Sozialisation ebenso vermeintlich unveränderbar in die Personen und damit auch in die Strukturen fest eingeschrieben sind (vgl. u. a. Galtung 1996, S. 9-46). Claudia Brunner (2020, S. 169) folgend, lässt Galtung damit aber ,,jene stets vorhandene Heterogenität und Dissidenz außer Acht [...], die er an anderer Stelle für seine Theoretisierung und Analyse globaler Gewalt- und Herrschaftsverhältnisse und vor allem für deren Überwindung als unverzichtbar erachtet“. Somit lassen sich auch hier erneut Unbestimmtheiten und Ambivalenzen konstatieren, die mit der starken normativen Aufladung des Konzepts verbunden sind und ohne ein differenziertes Beobachter*innenkonzept schwerlich zu lösen sind.
} 
nen im Sozialgefüge angesehen werden, sodass letztlich keine Gewalt im Sinne von violentia vorherrscht, sondern die Existenz von Machtstrukturen und sozialen Ungerechtigkeiten bzw. Ungleichheiten im Sinne der potestas beschrieben wird (vgl. Lindemann 2015, S. 507). Somit kann auch bei kultureller Gewalt wie bei struktureller Gewalt bzgl. der Reintegrationsmöglichkeit nicht von einer mit den neueren Ansätzen der Gewaltforschungen wirklich vergleichbaren Basis gesprochen werden. Die eigentliche Zuschreibung von Gewalt jenseits von potestas und deren kommunikativer Akt kann nur (reflexiv) durch ein/e/n außenstehende/s/n Dritte/s/n vorgenommen werden, was in Galtungs (1969, 1975a, S. 11-15, 1978a, S. 52-61, 1990, 1993b, 1996) Konzeptualisierung von struktureller, kultureller und personaler Gewalt im Rahmen rein dyadischer Täter*innen-Opfer-Konstellationen sowie im Kontext der strikten Zweiteilung des Weltpunktraums mit dem expliziten Komplikationen vermeidenden Ausschluss einer/s Dritten (tertium non datur; vgl. Galtung 1978a, S. 56) nicht möglich ist und damit auch dem neueren Ansätzen inhärenten zentralen Aspekt des kommunikativen Charakters von Gewalt keine Rechnung trägt. Und obgleich sich Galtung (1996, S. 16-18) mit der Hinwendung zum Buddhismus von dieser strikt dichotomen Perspektive distanziert und für eine Sowohl-als-auchSichtweise (im Sinne eines eingeschlossenen Dritten und einer doppelten Dialektik) plädiert, um der prozessualen Komplexität von Frieden und Gewalt Rechnung zu tragen, spezifiziert er die Beobachter*innenfrage in diesem Zuge nicht weiter.

Zur Lösung des bei Galtung (1975a, 1990, 1993b, 1996) unbestimmten (de-)legitimierenden Beobachter*innenproblems, schlägt Imbusch (2017a, S. 46-47) die Konzeptualisierung aktiver (politische Öffentlichkeit, öffentliche Meinung, Massenmedien) wie passiver (Normen, Gesetze, Recht) struktureller Dritter vor. Mit dieser Erweiterung, so Imbusch (2017a, S. 45-46), ließe sich zugleich ,unweigerlich [...] die Bedeutung struktureller Gewalt“ (Imbusch 2017a, S. 45) vor allem in der bis dato stets kritisierten „Analyse mikrosozialer Gewalt nachweisen“ (Imbusch 2017a, S. 46), da ,,aktive wie passive strukturelle Dritte einen Deutungs- und Legitimationsrahmen für Gewalt [liefern], den man selbst als Form struktureller Gewalt begreifen kann“ (Imbusch 2017a, S. 46).

Mit Blick auf den Deutungsrahmen scheint die über aktive strukturelle Dritte vermittelte und wahrgenommene strukturelle Gewalt dabei auf den ersten Blick durchaus fruchtbar - zumindest bzgl. der hierdurch eingeführten potenziellen Berücksichtigung einer/s externen und situativ relativierenden Beobachter*in/s in Galtungs Konzept. Dies würde in theoretischer Hinsicht auch die Möglichkeit eröffnen, die zuvor angesprochene, weiterhin existente Fokussierung auf erleidende Körper zu integrieren: Steht auch in Schroers (2004, S. 166-169) Beispiel der multiexkludierten Personen die Reduktion auf den leidensbasierten Körper weiterhin im Fokus, wird das Leiden der Betroffenen über den kommunikativen Akt der Anwendung personaler Gewalt sichtbar gemacht - und zwar über massenmediale Berichterstattungen bzgl. offen dargestellter personaler Gewalttaten (Aufstände, Revolten etc.). Über die mediale Berichterstattung und die Wahrnehmung der ebenfalls im Bereich aktiver struktureller Dritter verorteten öffentlichen Meinung könnte damit der Blick auf strukturelle Gewalt gelenkt werden, sodass diese wiederum erklärungsrelevanten Charakter im Kontext der beobachteten Mikrogewaltakte erhalten könnte. 
Trotz der durchaus überzeugend dargelegten Reintegrationsmöglichkeiten müssen hier dennoch zwei Aspekte bzgl. des analytischen Mehrwerts kritisch hinterfragt werden: Zum einen scheint das Konzept der strukturellen Dritten den bereits in neueren gewaltsoziologischen Ansätzen implementierten und detailliert ausgearbeiteten (institutionellen oder organisationalen) Dritten zu entsprechen, sodass der Mehrwert hier fraglich erscheint. Hinzu kommt, dass die Existenz struktureller Gewalt zwar durch aktive strukturelle Dritte beobachtet und aufgedeckt werden kann und wird, der Fokus dieser Beobachtung - und damit auch der kommunikative Akt von Gewalt - aber, wie zuvor aufgezeigt, weiterhin auf erleidenden und Gewalt erfahrenden Körpern liegt. Zum anderen muss die Erweiterung um strukturelle Dritte auch dem vorher angeführten Aspekt Rechnung tragen, dass diese jeweils ihren eigenen Beobachtungsschemata mit spezifischen und potenziell divergierenden sozialen Normen und individuellen Werten unterliegen, was mit der situativen Frage nach der (De-)Legitimation von Gewalt einhergeht (vgl. Koloma Beck 2011, S. 351; Lindemann 2017).

Diese spezifischen und potenziell divergierenden Normen und sozialen Werte, „die als Denk- und Handlungsweisungen eines Gemeinwesens aufgefasst werden können, die von der Gesellschaft insgesamt oder von den staatlichen Institutionen getragen werden“ (Imbusch 2017a, S. 46) sowie den damit verbundenen (de-)legitimierenden Aspekt von Gewalt verortet Imbusch (2017a, S. 46) dabei im Bereich der passiven strukturellen Dritten, was wiederum mit der basalen (de-)legitimierenden kulturellen Gewalt in Galtungs (1975a, 1990, 1993b) Gewaltdreieck verbunden ist. Das Problem, das mit diesen Legitimationsformen im Sinne der kulturellen Gewalt sowie mit Blick auf deren Konzeptualisierung als passive strukturelle Dritte verbunden ist, zeigt sich erneut mit Blick auf die analytische Trennung von struktureller und kultureller Gewalt sowie der eingangs angesprochenen unbestimmten Abgrenzung von Macht und Gewalt. Auf Ersteres verweist Imbusch (2017a, S. 46) selbst, wenn er darauf aufmerksam macht, dass es bei den passiven strukturellen Dritten als

Delegitimationsfunktionen von Gewalt und [...] [dem; AB] strukturellen Rahmen, in dem Gewalt [...] verboten, erlaubt oder sogar geboten ist [...] noch einer gewissen begrifflichen Anstrengung bedarf, um die Formen struktureller Gewalt von jenen der kulturellen Gewalt möglichst trennscharf abzugrenzen. (Imbusch 2017a, S. 46)

Während hier also durchaus noch Potenzial für künftige Weiterentwicklungen vorhanden ist, besteht das Grundproblem weiterhin: Kulturelle Gewalt spiegelt keine Gewalt im Sinne der violentia wieder, sondern ist aufgrund ihrer zirkulären Verwobenheit mit struktureller Gewalt und der Tatsache einer zeitlichen Invarianz als (Aspekt von) Macht im Sinne der potestas zu sehen (vgl. Galtung 1975a, S. 20-23, 1993b, S. 57-59): Kulturelle Gewalt legitimiert strukturelle Gewalt und zur Aufrechterhaltung der bestehenden ungleichen Verteilungsmuster reproduziert Letztere wiederum jene Legitimationsbasis über die strukturellen Gewaltmechanismen (Galtung 1975a, S. 20-23, 1993b, S. 57-59), sodass es sich bei kultureller Gewalt einerseits um ein Mittel zur Machtherstellung und -sicherung handelt. Andererseits zeigt sich aber auch ein nicht situationsgebundener, zeitlich mittelbarer (sogar in- 
varianter) Zusammenhang, der über strukturelle Reproduktionen institutionalisiert wird und hierarchisch gegliederte überpersonale Positionsgefüge integriert (auserwählt/nicht-auserwählt, wir/andere, westlich/nicht-westlich, wahr/nicht-wahr), was, wie bei struktureller Gewalt, ebenfalls auf potestas und nicht auf violentia verweist. ${ }^{26}$

Betrachtet man z.B. den von Galtung (1990, 1993b) angeführten Aspekt der Religion, so ist deren gewaltlegitimierende Basis empirisch nicht von der Hand zu weisen, was u. a. die vielzähligen Kriege und terroristischen Attentate, die im Namen eines Gottes seit den Kreuzzügen, dem Dreißigjährigen Krieg oder in Form islamistisch motivierter Terrorakte ausgeführt wurden und werden, verdeutlichen. Dies gilt im Übrigen auch für den von Galtung $(1985,1993 a)$ paradigmatisch für die Schaffung von (Welt-)Frieden herangezogenen Buddhismus, der keineswegs gänzlich als gewaltfrei zu werten ist (vgl. Elison 1973; Jerryson und Jurgensmeyer 2010), sodass Galtung hier selbst einer westlich verklärten Perspektive zu verfallen scheint. Aufgrund der funktions- und strukturbezogenen personalen (physischen) Gewalteinwirkungen auf den Schlachtfeldern, Kriegsschauplätzen und situativen Tatorten, geht die ausgeübte Gewalt aber mit dem körperlichen Erleiden und den Schmerzen der Opfer einher. Dabei ist der eigentliche legitimierende Akt nicht nur in Machtund Herrschaftsstrukturen eingebunden, sondern fester Bestandteil dessen, da er mit dem Gehorsam der Befehlsausführung einhergeht und als Mittel zur Machtsicherung reproduziert wird, was somit der auf institutionalisierter Macht beruhenden traditionellen Herrschaft, ,,auf Grund der Heiligkeit altüberkommener (,von jeher bestehender') Ordnungen“ (Weber 1980, S. 130), entspricht (vgl. Weber 1980, S. 130-140). Dies zeigt sich in ähnlicher Weise auch bei den ideologischen (nationalstaatlichen), auf der Abgrenzung westlich/nicht-westlich beruhenden und der durch die Logik der Wissenschaften legitimierten anderen kulturellen Gewaltformen: Unter Rekurs auf Bauman (1992, S. 33-71, 2002, S. 98-131) entsprechen diese Legitimationsvarianten - im Sinne (re)produzierter klassischer Freund/Feind Dichotomien - der typischen (aufbauenden wie erhaltenden) Ordnungslogik moderner Gesellschaften und sind somit ebenso unmittelbar in und mit Macht- und Herrschaftsstrukturen verbunden, zu deren Durchsetzung ein hierarchisch strukturierter und überpersonaler Staatsapparat benötigt wird. Dessen idealtypische Form kann in Webers (1980, S. 124-130) - gleichsam auf institutionalisierter Macht beruhender - bürokratischer Herrschaft gesehen werden, die sich ebenfalls durch Gehorsam gegenüber dem nationalstaatlichen Befehlsgeber auszeichnet und „Herrschaft kraft Wissen“ (Weber 1980, S. 128) sichert. Aufgrund der Konstruktion dieser Unterscheidungen durch den Herrschaftsapparat als ordnungsstiftendem „Gärtner“ (Bauman 1992, S. 35), wirken diese Dimensionen kultureller Gewalt bzgl. physisch verletzender Gewalt zwar legitimierend, bezogen auf ihre eigene Wirkweise sind sie aber untrennbar mit dem grundlegenden strukturellen Machtkontext reproduktiv verbunden (vgl. Bauman 1992, S. 33-71, 2002, S. 98-131). Anstatt um Gewalt im Sinne von violentia handelt es sich somit ebenfalls um zeitlich mittelbare potestas.

\footnotetext{
${ }^{26}$ Dies kann zudem auf die (in Teilen tautologische) argumentative Zirkularität zurückgeführt werden, welche in analytischer Hinsicht nicht aufgebrochen werden kann, sodass eine differenzierte Betrachtung von struktureller Gewalt als Macht im Sinne von potestas und kultureller Gewalt im Sinne von violentia aufgrund der galtungschen Erklärungsstruktur nicht möglich ist.
} 
Der analytische Mehrwert der Integration auf kultureller Gewalt beruhender struktureller Gewalt ist somit auch in diesem Punkt fraglich. Die Unbestimmtheit und Undifferenziertheit des Konzepts bleibt nicht nur weiterhin bestehen, sondern wird über die Einbeziehung struktureller Dritter zudem noch verkompliziert bzw. noch unbestimmter. Vor allem, da hier auch eher eine umgekehrte Ergänzung vorzuliegen zu scheint: Statt Galtungs (1975a, 1990, 1993b) Ansatz für die neuere Gewaltforschung fruchtbar zu machen, weist die von Imbusch (2017a) vorgenommene Ergänzung um strukturelle Dritte gerade darauf hin, diesen um Elemente der neueren Gewaltforschung zu ergänzen, um dessen Erklärungspotenzial analytisch zu schärfen. Mit anderen Worten kann dies als Versuch gelesen werden, gerade jene (erneute) Unbestimmtheit der methodologisch zweiwertigen Einteilung des Weltpunktraums um ein Konzept zu erweitern, welches der Sowohl-als-auch-Perspektive gerecht wird und dabei die Frage nach dem Beobachter*innenmodus dezidiert in den Blick nimmt. Was im Kontext des galtungschen Konzepts aufgrund der aufgezeigten Probleme und Unbestimmtheiten nicht gegeben ist, könnte somit gerade mithilfe neuerer gewaltsoziologischer Ansätze fruchtbar integriert und umgesetzt werden. Jenseits starrer Täter*innen-Opfer-Dichotomien vermögen triadisch-prozessuale Perspektiven, ohne (moralisch) normativ aufgeladene Konzeptualisierung, (feldabhängig) reflexiv zu bestimmen, was als Gewalt im Kontext gültiger (Verfahrens-)Ordnungen gilt und was nicht (vgl. u. a. Hoebel und Knöbl 2019; Koloma Beck 2017; Lindemann 2017). Eine solche beobachter*innenabhängige Perspektive scheint für eine an Galtung orientierte Friedens- und Konfliktforschung äußert gewinnbringend. Denn rekonstruiert man ausgehend vom empirischen Gegenstand und dessen sozio-kulturell variabler Einbettung, ob hier aufseiten der Opfer tatsächlich empfundene Gewalt vorliegt und distanziert man sich damit (selbstreflexiv) zugleich von einer mit der Auffassung von modernen (Welt-)Gesellschaften verbundenen spezifisch westlich-normativen Sichtweise, dann vermeidet man gewissermaßen sowohl jene hiermit verbundenen Gewaltapologetiken im Sinne der traditionellen Modernität-Barbarei Differenzierung als auch mit Blick auf die Herstellung von und die Frage nach Frieden(ssicherung) eine hieran angelehnte widerwillige Zivilisierung Anderer auf Basis eines zivilisations-hexagonischen eurozentristischen Blicks (vgl. Brunner 2020; Elias 1978; Koloma Beck 2011, 2017, 2019; Lindemann 2014, 2017, 2018; Senghaas 1995).

Das analytisch instruktive Potenzial einer solch triadisch angelegten Perspektive für die Friedens- und Konfliktforschung haben u.a. bereits Teresa Koloma Beck und Tobias Werron $(2013$, 2018) bzgl. globaler Gewaltmärkte aufgezeigt, wenn sie konstatieren, dass die sozialen Dynamiken von Gewalt erst in einer triadischen Beziehung evolvieren, die die drei Erfahrungsmodi

(a) Leid verursachende Täter*in;

(b) Leid erfahrenes Opfer und

(c) diesen Akt als Gewalt identifizierende Beobachter*in verbindet (vgl. Kolo-

ma Beck und Werron 2018, S. 280).

Dies ist vor allem dann besonders fruchtbar, ,when it comes to analyzing violence in the social formation we call Modernity, considering the increasingly gener- 
alized normative aversion against the use of force" (Koloma Beck und Werron 2018, S. 280).

The institutionalization of ideals of the value of life has created a paradoxical situation, in which there is a strong and generalized normative rejection of the use of force against other human beings, but at the same time still a need to - at least sometimes - use force to maintain and defend the very social order that cherishes these ideals. (Koloma Beck und Werron 2018, S. 280-281)

Zudem kann auch die Einbindung des bei Galtung ebenfalls vorhandenen Körperbezugs ein fruchtbarer Aspekt für die Friedens- und Konfliktforschung sein. Gerade mit Blick auf die bei Galtung erwähnten therapeutischen Mittel scheint zumindest Potenzial vorhanden. Galtung (1996, S. 27-37) verweist bzgl. der Therapie zwar auf die Möglichkeiten einer Schulung und Weiterbildung sowohl fachspezifischen (politischen) Personals als auch in genereller (gewissermaßen aufklärerischer) Hinsicht, lässt aber jenseits des Materialeinsatzes von Friedens- und Gewaltheorien offen, wie der tatsächlich therapeutisch interventive Aspekt umgesetzt werden kann. Ein Fokus auf die Bedeutung körperlicher Akteur*innen kann hierbei von Nutzen sein, da Galtung (1996, S. 37-39) schließlich ebenso auf das Potenzial der Verbindung von Körper-Geist-Struktur-Kultur hinweist, eine theoretische Präzisierung aber offenlässt. Die Bedeutung körperlicher Akteur*innen und damit auch die Integration einer sozialtheoretischen Komponente kann in Verbindung mit triadischen Konzeptualisierungen somit helfen genau jenen Aspekt analytisch zu schärfen. Die Mittel zur Therapie liegen somit jenseits der diagnostizierten und prognostizierten strukturellen wie kulturellen Problematiken auf der Ebene der Akteur*innen, die als handlungsfähige Träger*innen die sozio-kulturellen Gebilde und Strukturen aufdecken und verändern können - dies würde dann auch politisch interventiv, z.B. in der Bedeutung sozialer Bewegungen verortet werden können. Ein hiermit verbundener, analytisch enger körperlicher Gewaltbegriff mag zwar kein politisches Potenzial aufweisen (vgl. Brock 1995, S. 344), kann aber letztlich über die in neueren Ansätzen der Gewaltsoziologie vorhandene triadische Konzeptualisierung und Berücksichtigung variabler sozio-kultureller Strukturen im Verlauf der Theoriekonstruktion additiv politisch fruchtbar gemacht werden (vgl. Brock 1995, S. 344; und u. a. Lindemann 2017). Der Rekurs auf sozialtheoretische Perspektiven in Galtungs Konzept kann zudem eine bestimmte Problematik der von Imbusch aufgeworfenen Weiterentwicklung der galtungschen Theorie umgehen. Nämlich jene, dass bei diesem Vorhaben und im Sinne der vom Imbusch angesprochenen aktiven wie passiven Dritten eine mindestens systemtheoretische Revitalisierung des galtungschen Konzeptes notwendig wäre - zumindest wenn man den erkenntnistheoretischen Entstehungskontext und den kommunikativen Aspekt in Galtungs Ansatz ernst nehmen will, wie dies bspw. Schroer (2004) in Anlehnung an die luhmannsche Theorie aufgezeigt hat. In der theoretischen Konsequenz weist dies aber die Problematik auf, dass Gewalt dann zwar als Kommunikation eingefangen und aufgefasst werden kann, der Status leidensfähiger Lebewesen aber bzgl. ihrer Funktion als psychische Systeme exkludiert werden müsse und zudem auch ein anders gelagerter Machtund Gewaltbegriff nebst der Reflexion bzgl. der Rolle und Funktion von Gewalt als parasitärem System von Nöten wäre (vgl. Baecker 2007, S. 29-63; Luhmann 1984, 
2012; Messmer 2003). Gegenüber einer solchen zu berücksichtigenden umfassenden theoretischen Rekonstruktion des galtungschen Konzepts scheint eine Reintegration neuerer gewaltsoziologischer Ansätze, vor allem auch bzgl. der explikativen und nicht nur problembeschreibenden Absicht, in analytischer Hinsicht besser integrierund nutzbar zu sein.

\section{Resümee}

Das Konzept der strukturellen Gewalt wird Imbusch (2017a) zufolge von der neueren gewaltsoziologischen Forschung unterschätzt. Vor diesem Hintergrund hat er für dessen Wiederbelebung und gewaltsoziologische Reintegration sowie für dessen kritisch gesellschaftsanalytisches Potenzial plädiert (vgl. Imbusch 2017a). Während strukturelle Gewalt möglicherweise ein tatsächlich „,unterschätzte[r] Begriff“ (Imbusch 2017a, S. 28) für die hier nicht weiter untersuchte kritische Gesellschaftstheorie sein kann, so muss dieses Plädoyer aus einer explizit neueren gewaltsoziologischen Perspektive aber einer kritischen Prüfung unterzogen werden. Ist hier doch gerade der analytische Mehrwert aufgrund der weiterhin existenten „Schwachstellen“ (Imbusch 2017a, S. 37) fraglich. Zumindest im Kontext des hier vorgenommenen einfachen Vergleichs mit Blick auf das geteilte Bezugsproblem Gewalt lässt sich der von Imbusch konstatierte Mehrwert für neuere Ansätze der Gewaltforschung nicht ohne Weiteres bestätigen. Das terminologische Grundproblem und die vorhandenen Abgrenzungsprobleme zwischen struktureller und physischer sowie struktureller und kultureller Gewalt durchziehen Galtungs (1969, 1975a, 1990, 1993b, 1996) gesamtes Konzept mit dem Ergebnis, dass im Kontext des galtungschen (1975a, 1990, 1993b) Gewaltdreiecks analytisch letztlich vornehmlich mit Machtaspekten (im Sinne von potestas) operiert wird. Da Galtungs Gewalttrias aber stets nur zusammen denk- und anwendbar ist und lediglich der Aspekt der physischen Gewalt einer als Schmerz und Leid verursachenden Gewalt im Sinne von violentia zuzuordnen ist, liegt für eine Reintegration in aktuelle Ansätze der Gewaltforschung somit - trotz gemeinsamen Bezugsproblems und einer erweiterten Sicht auf soziale Kontextfaktoren keine analytisch umfängliche Vergleichs- und Integrationsbasis vor (vgl. Greshoff et al. 2007, S. 9-10). Wie im Gewaltdiskurs der 1990er-Jahre und der immer wiederkehrenden Debatte um die Frage „Körper oder Struktur?“ (Braun 2019a) werden somit auch bei Imbuschs Vorschlag zwar ,mit ähnlichen Wortgebräuchen“ (Greshoff et al. 2007, S. 9) operierende Konzepte in den Blick genommen, diese beziehen sich aber in analytischer Hinsicht auf grundlegend unterschiedliche Positionen. In theoretisch einfach vergleichender Hinsicht handelt es sich hierbei um eine „Scheinkontroverse[.] oder ein unsinnige[s; AB] gegeneinander Ausspielen von verschiedenen Positionen“ (Greshoff et al. 2007, S. 9-10). Dabei übersieht Imbusch auch, dass die nicht mehr zeitgemäße ,fortwährende Polarisierung von körper- und strukturbezogenen Ansätzen“ (Braun 2019a, S. 71) von neueren gewaltsoziologischen Ansätzen nicht mehr geteilt, sondern bereits konzeptuell integrativ behandelt wird. Vor diesem Hintergrund lässt sich damit auch kein analytischer Mehrwert hinsichtlich der Wiederbelebung des Konzepts der strukturellen Gewalt konstatieren: Das aufgezeigte Potenzial mit Blick auf die Berücksichtigung (sozial-)struktureller Bedingungen 
und Hintergrundfaktoren zur Erklärung von Gewalt ist im Kontext aktueller Ansätze kein Novum, sondern wird bereits in prozessualen und triadisch angelegten Konzepten berücksichtigt - und zwar ohne normative Aufladung und einer definiten dyadischen Täter*innen-Opfer-Konstellation (vgl. Galtung 1975a). ${ }^{27}$ Darüber hinaus kennzeichnen sich aktuelle Ansätze der Gewaltforschung auch gerade durch eine forschungspraktische, analytisch trennscharfe Betrachtung von Macht und Gewalt, was im Kontext des aufgezeigten Grund- und der weiteren Abgrenzungsprobleme bei Galtung (1975a, 1990, 1993b) nicht ermöglicht werden kann. Eine konzeptuelle Engführung auf körperliche Gewalt mag zwar kein politisch interventives Potenzial aufweisen, ist aber dennoch in der Lage, ein solches Interventionspotenzial - unter Berücksichtigung der Komplexität von Gewalt - additiv im Zuge der Forschung zu generieren. Hinsichtlich der Frage nach dem analytischen Mehrwert verweisen demgegenüber gerade neuere gewaltsoziologische Ansätze auf das fruchtbare Integrationspotenzial für die galtungsche Friedens- und Konfliktforschung. Gerade der Rekurs auf körperliche Gewalt und eine hieran anschließende triadische Konzeptualisierung eines Beobachter*innenkonzeptes scheinen zumindest insofern gewinnbringend, als hiermit etwaige blinde Flecken und (eurozentristische) Sichtweisen im Sinne des modernen (westlichen) Zivilisationscredos auf die Frage nach der Herstellung von Frieden in den Blick genommen und kritisch (selbst-)reflexiv hinterfragt werden können - auch im Kontext einer globalen Weltgesellschaft.

Vor diesem Hintergrund lässt sich somit aus einer explizit neueren gewaltsoziologischen Perspektive abschließend konstatieren, dass es sich bei struktureller Gewalt diesbezüglich um einen analytisch überschätzten und keinen ,unterschätzten Begriff“ (Imbusch 2017a, S. 28) handelt.

Funding Open Access funding enabled and organized by Projekt DEAL.

Open Access Dieser Artikel wird unter der Creative Commons Namensnennung 4.0 International Lizenz veröffentlicht, welche die Nutzung, Vervielfältigung, Bearbeitung, Verbreitung und Wiedergabe in jeglichem Medium und Format erlaubt, sofern Sie den/die ursprünglichen Autor(en) und die Quelle ordnungsgemäß nennen, einen Link zur Creative Commons Lizenz beifügen und angeben, ob Änderungen vorgenommen wurden.

Die in diesem Artikel enthaltenen Bilder und sonstiges Drittmaterial unterliegen ebenfalls der genannten Creative Commons Lizenz, sofern sich aus der Abbildungslegende nichts anderes ergibt. Sofern das betreffende Material nicht unter der genannten Creative Commons Lizenz steht und die betreffende Handlung nicht nach gesetzlichen Vorschriften erlaubt ist, ist für die oben aufgeführten Weiterverwendungen des Materials die Einwilligung des jeweiligen Rechteinhabers einzuholen.

Weitere Details zur Lizenz entnehmen Sie bitte der Lizenzinformation auf http://creativecommons.org/ licenses/by/4.0/deed.de.

27 Vgl. Armbruster (2016); Braun (2016); Hoebel (2014); Koloma Beck (2011, 2015, 2017); Leuschner (2016); Lindemann (2014, 2015, 2017); Nassauer (2015, 2019); Reemtsma (2009); Sutterlüty (2015); Wieviorka $(2006,2014)$. 


\section{Literatur}

Abbott, Andrew. 2004. Methods of discovery. New York: NY.

Anicker, Fabian. 2017. Theorienvergleich als methodologischer Standard der soziologischen Theorie. Zeitschrift für Soziologie 46(2):71-88.

Arendt, Hannah. 1970. Macht und Gewalt. München: Piper.

Armbruster, André. 2016. Soziale Unterstützung für Gewalt in Interaktionen. Situativ erzeugte Erwartungen zur Ermöglichung von Gewalt am Fall des Soweto-Aufstands 1976. In Situationen der Gewalt, Hrsg. Claudia Equit, Axel Groenemeyer, und Holger Schmidt, 361-378. Weinheim: Beltz Juventa.

Baberowski, Jörg. 2008. Gewalt verstehen. Zeithistorische Forschungen 5:5-17.

Baecker, Dirk. 2007. Wozu Gesellschaft? Berlin: Kadmos.

Bauman, Zygmunt. 1992. Moderne und Ambivalenz. Hamburg: Hamburger Edition.

Bauman, Zygmunt. 2002. Dialektik der Ordnung. Hamburg: EVA.

Bauman, Zygmunt. 2003. Flüchtige Moderne. Frankfurt a. M.: Suhrkamp.

Bauman, Zygmunt, und David Lyon. 2013. Daten, Drohnen, Disziplin. Berlin: Suhrkamp.

Berger, Peter L., und Thomas Luckmann. 2000. Die gesellschaftliche Konstruktion der Wirklichkeit, 17. Aufl., Frankfurt a. M.: Fischer.

Bourdieu, Pierre. 1993. Sozialer Sinn. Frankfurt a. M.: Suhrkamp.

Bourdieu, Pierre. 1997. Eine sanfte Gewalt. Pierre Bourdieu im Gespräch mit Irene Dölling und Margareta Steichrücke. In Ein alltägliches Spiel - Geschlechterkonstruktionen in der sozialen Praxis, Hrsg. Irene Dölling, Beate Krais, 218-230. Fankfurt a. M: Suhrkamp.

Bourdieu, Pierre. 2005. Die männliche Herrschaft. Frankfurt a. M.: Suhrkamp.

Braun, Andreas. 2015. Campus shootings. Bielefeld: transcript.

Braun, Andreas. 2016. Zielgerichtete Gewalt zwischen Situation und Identität. School Shootings als identitätsbehauptende Gewaltsituation. In Situationen der Gewalt, Hrsg. Claudia Equit, Axel Groenemeyer, und Holger Schmidt, 246-261. Weinheim: Beltz Juventa.

Braun, Andreas. 2019a. Zur Entdeckung verkörperter Gewalt. Zeitschrift für Theoretische Soziologie 8(1):63-73.

Braun, Andreas. 2019b. Interpersonal violence as an intrinsic part of the civilizing process. European Journal of Sociology 60(2):283-312.

Braun, Andreas. 2020. Gewaltsoziologie im Wandel. Über Situationen, Prozesse und Ordnungen als Bestandteile zur Erforschung von Gewalt. Österreichische Zeitschrift für Soziologie 45(S1):5-21.

Brock, Lothar. 1995. Friedensforschung im Zeichen immer neuer Kriege. In Frieden als Zivilisierungsprojekt, Hrsg. Wolfgang R. Vogt, 340-350. Baden-Baden: Nomos.

Brunner, Claudia. 2020. Epistemische Gewalt. Bielefeld: transcript.

Collins, Randall. 1975. Conflict sociology. New York: Academic Press.

Collins, Randall. 2011. Dynamik der Gewalt. Hamburg: Hamburger Edition.

Collins, Randall. 2019. An der Schwelle zur Gewalt. Mittelweg 36 28(1-2):60-69.

Crowther, Jonathan. 1995. Oxford advanced learner's dictionary of current English, 5. Aufl., Oxford: Oxford University Press.

De Maio, Fernando, und David Ansell. 2018. "as natural as the air around us": on the origin and development of the concept of structural violence in health research. International Journal of Health Services 48(4):749-759.

Der-Ian Yeh, Theresa. 2006. The way to peace: a buddhist perspective. International Journal of Peace Studies 11(1):91-112.

Elias, Norbert. 1978. Über den Prozeß der Zivilisation. Bd. 1, 2. Frankfurt a. M.: Suhrkamp.

Elison, George. 1973. Deus destroyed: the image of christianity in early modern Japan. Cambridge: Harvard University Press.

Equit, Claudia, und Holger Schmidt. 2016. Theoretische Rahmung von (Gewalt-)Situationen. Implikationen für die Forschung. In Situationen der Gewalt, Hrsg. Claudia Equit, Axel Groenemeyer, und Holger Schmidt, 40-64. Weinheim: Beltz Juventa.

Fujii, Lee Ann. 2009. Killing neighbors. Ithaca: Cornell University Press.

Fujii, Lee Ann. 2013. The puzzle of extra-lethal violence. Perspectives on Politics 11(2):410-426.

Galtung, Johan. 1969. Violence, peace, and peace research. Journal of Peace Research 6(3):167-191.

Galtung, Johan. 1971. A structural theory of imperialism. Journal of Peace Research 8(2):81-117.

Galtung, Johan. 1974a. World indicators. https://www.transcend.org/galtung/papers/World\%20Indicators. pdf;. Zugegriffen: 16. Sept. 2019. 
Galtung, Johan. 1974b. Is peaceful research possible? On the methodology of peace research. https://www. transcend.org/galtung/papers/Is\%20Peace\%20Research\%20Possible\%20-\%200n\%20the\%20Metho dology\%20of\%20Peace\%20Research.pdf;. Zugegriffen: 16. Sept. 2019.

Galtung, Johan. 1975a. Strukturelle Gewalt. Reinbek bei Hamburg: Rowohlt.

Galtung, Johan. 1975b. The specific contribution of peace research to the study of the causes of violence-typologies. https://www.transcend.org/galtung/papers/The\%20Specific\%20Contribution $\% 20$ of $\% 20$ Peace $\% 20$ Research $\% 20$ to $\% 20$ the $\% 20$ Study $\% 20$ of $\% 20$ the $\% 20$ Causes $\% 20$ of $\% 20$ Violence \%20-\%20Typologies.pdf;. Zugegriffen: 16. Sept. 2019.

Galtung, Johan. 1978a. Methodologie und Ideologie. Frankfurt a. M.: Suhrkamp.

Galtung, Johan. 1978b. On violence in general and terrorism in particular (paper prepared for the new zealand law society convention, Auckland, 31 march 1978). https://www.transcend.org/galtung/papers/ On $\% 20$ Violence $\% 20$ in $\% 20$ General $\% 20$ and $\% 20$ Terrorism $\% 20$ in $\% 20$ Particular.pdf;. $\quad$ Zugegriffen: 16. Sept. 2019.

Galtung, Johan. 1983. Struktur, Kultur und intellektueller Stil: Ein vergleichender Essay über sachsonische, teutonische, gallische und nipponische Wissenschaft. Leviathan 11(3):303-338.

Galtung, Johan. 1985. Peace and buddhism. https://www.transcend.org/galtung/papers/Peace\%20and \%20Buddhism.pdf; Zugegriffen: 6. Juli 2020.

Galtung, Johan. 1990. Cultural violence. Journal of Peace Research 27(3):291-305.

Galtung, Johan. 1993a. Buddhism, a quest for unity and peace. Sri Lanka: Sarvodaya.

Galtung, Johan. 1993b. Kulturelle Gewalt. Zur direkten und strukturellen Gewalt tritt die kulturelle Gewalt. In Aggression und Gewalt, Hrsg. Hans-Peter Nolting, Hans-Georg Wehling, 52-73. Stuttgart: Kohlhammer.

Galtung, Johan. 1996. Peace by peaceful means. London.: Sage.

Giddens, Anthony. 1984. The constitution of society. Cambridge: Polity Press.

Geser, Hans. 1990. Organisationen als soziale Akteure. Zeitschrift für Soziologie 19(6):401-417.

Göhler, Gerhard. 1997. Wie verändern sich Institutionen? Revolutionärer und schleichender Institutionenwandel. In Institutionenwandel, Hrsg. Gerhard Göhler, 21-56. Opladen: Westdeutscher Verlag.

Gresshoff, Rainer. 1999. Lassen sich die Konzepte von Max Weber und Niklas Luhmann unter dem Aspekt „Struktur und Ereignis“ miteinander vermitteln? In Struktur und Ereignis in theorievergleichender Perspektive, Hrsg. Rainer Gresshoff, Georg Kneer, 13-50. Opladen: Westdeutscher Verlag.

Greshoff, Rainer, Gesa Lindemann, und Uwe Schimank. 2007. Theorienvergleich und Theorieintegration - Disziplingeschichtliche und methodische Überlegungen zur Entwicklung eines paradigmenvermittelnden ,,conceptual framework“ für die Soziologie. https://uol.de/fileadmin/user_upload/sowi/ag/ast/ download/dp/ast-dp-1-07.pdf;. Zugegriffen: 16. Sept. 2019.

Gugutzer, Robert. 2004. Soziologie des Körpers. Bielefeld: transcript.

Gukenbiehl, Hermann L. 2002. Institution und Organisation. In Einführung in Hauptbegriffe der Soziologie, Hrsg. Hermann Korte, Bernhard Schäfers, 143-159. Opladen: Leske + Budrich.

Hartmann, Eddie, 2017. Gewalt als sozialer Tatbestand. Grundzüge einer Allgemeinen Soziologie der Gewalt im Anschluss an Jan Philipp Reemtsma (Diskussionspapier zur Tagung Streitfragen der soziologischen Gewaltforschung, Aachen, 17./18. Nov. 2017)

Hamer, Jennifer F., und Clarence Lang. 2015. Race, structural violence, and the neoliberal university: the challenges of inhabitation. Critical Sociology 41(6):897-912.

Hauffe, Tobias, und Thomas Hoebel. 2017. Dynamiken soziologischer Gewaltforschung. Soziologische Revue 40(3):369-384.

Hodgetts, Darrin, Kerry Chamberlain, Shiloh Groot, und Yardena Tankel. 2013. Urban poverty, structural violence and welfare provision for 100 families in Auckland. Urban Studies 51(10):2036-2051.

Hoebel, Thomas. 2014. Organisierte Plötzlichkeit. Eine prozesssoziologische Erklärung antisymmetrischer Gewaltsituationen. Zeitschrift für Soziologie 43(6):441-457.

Hoebel, Thomas, und Hartmann Eddie. 2020. Die Schweigsamkeit der Gewalt durchbrechen. WestEnd 17(1):71-79.

Hoebel, Thomas, und Wolfgang Knöbl. 2019. Gewalt erklären! Hamburg: Hamburger Edition.

Hoebel, Thomas, und Teresa Koloma Beck. 2019. Theorizing Violence. Über die Indexikalität von Gewalt und ihrer soziologischen Analyse. Zeitschrift für Theoretische Soziologie 8(1):4-11.

Hoebel, Thomas, und Stefan Malthaner (Hrsg.). 2019. Im Brennglas der Situation. Neue Ansätze in der Gewaltsoziologie. Hamburg: Hamburger Edition.

Hradil, Stefan. 2016. Soziale Ungleichheit, soziale Schichtung und Mobilität. In Einführung in die Hauptbegriffe der Soziologie, Hrsg. Hermann Korte, Bernhard Schäfers, 247-275. Wiesbaden: Springer.

Imbusch, Peter. 2000. Gewalt - Stochern in unübersichtlichem Gelände. Mittelweg 36 9(2):24-40. 
Imbusch, Peter. 2002. Der Gewaltbegriff. In Internationales Handbuch der Gewaltforschung, Hrsg. Wilhem Heitmeyer, John Hagan, 26-57. Wiesbaden: Westdeutscher Verlag.

Imbusch, Peter. 2004. Mainstreamer' versus ,Innovateure' der Gewaltforschung: Eine kuriose Debatte. In Gewalt, Hrsg. Wilhelm Heitmeyer, Hans-Georg Soeffner, 125-148. Frankfurt a. M.: Suhrkamp.

Imbusch, Peter. 2005. Moderne und Gewalt. Wiesbaden: VS.

Imbusch, Peter. 2017a. Strukturelle Gewalt. Plädoyer für einen unterschätzten Begriff. Mittelweg 36 26(3):28-51.

Imbusch, Peter. 2017b. Die Rolle von ,Dritten'. Eine unterbelichtete Dimension von Gewalt. In Zwischen Tätern und Opfern, Hrsg. Philipp Batelka, Michael Weise, und Stephanie Zenhle, 47-74. Göttingen: Vandenhoeck \& Ruprecht.

Inhetveen, Katharina. 2017. Gewalt. In Handbuch Körpersoziologie, Bd. 2, Hrsg. Robert Gugutzer, Gabriele Klein, und Michael Meuser, 101-115. Wiesbaden: Springer.

Jaberg, Sabine. 2019. Die kulturelle Dimension von Gewalt und Frieden bei Johan Galtung. In Kulturelle Vielfalt als Dimension des gerechten Friedens, Hrsg. Sarah Jäger, André Munzinger, 19-45. Wiesbaden: Springer.

Jerryson, Michael K., und Mark Jurgensmeyer (Hrsg.). 2010. Buddhist warfare. Oxford: Oxford University Press.

Katz, Jack. 1988. Seductions of crime. New York: Basic Books.

Katz, Jack. 2020. Seduction, the street and emotion. Bingley: Emerald.

Kern, Leslie. 2015. Rhythms of gentrification: eventfulness and slow violence in a happening neighbourhood. Cultural Geographies 23(3):441-457.

Kersten, Anne. 2020. Eigensinnigkeiten häuslicher Gewalt. Eine netzwerktheoretische Perspektive. WestEnd 17(1):81-92.

Knöbl, Wolfgang. 2019. Collins im Kontext. Mittelweg 36 28(1-2):15-39.

Koepp, Tabea, und Chris Schattka. 2020. Eine kuriose Debatte wiederholt sich. Österreichische Zeitschrift für Soziologie 45(S1):23-42.

Koloma Beck, Teresa. 2011. The eye of the beholder: violence as a social process. International Journal of Conflict and Violence 5:345-356.

Koloma Beck, Teresa. 2015. Sozialwissenschaftliche Gewalttheorie heute: Sechs Thesen. https://soziopolis. de/beobachten/gesellschaft/artikel/sozialwissenschaftliche-gewalttheorie-heute/;. Zugegriffen: 16. Sept. 2019.

Koloma Beck, Teresa. 2017. Gewalt als leibliche Erfahrung. Ein Gespräch mit Teresa Koloma Beck. Mittelweg 36 26(3):52-73.

Koloma Beck, Teresa. 2019. Welterzeugung. Gewaltsoziologie als kritische Gesellschaftstheorie. Zeitschrift für Theorietische Soziologie 8(1):12-23.

Koloma Beck, Teresa, und Tobias Werron. 2013. Gewaltwettbewerbe. Gewalt in globalen Konkurrenzen um Aufmerksamkeit und Legitimität. Leviathan 27:249-277.

Koloma Beck, Teresa, und Tobias Werron. 2018. Violent conflictition. Armed conflicts and global competition for attention and legitimacy. International Journal of Politics, Culture, and Society 31(3):275-296.

Kron, Thomas. 2019. Gewalt als komplexes Phänomen. https://publikationen.soziologie.de/index.php/ kongressband_2018/article/view/974/1214;. Zugegriffen: 7. Juli 2020.

Kron, Thomas. 2020. Gewalt und emotionale Energie. Österreichische Zeitschrift für Soziologie 45(S1): 113-134.

Lepsius, M. Rainer. 1995. Institutionenanalyse und Institutionenpolitik. In Politische Institutionen im Wandel, Hrsg. Birgitta Nedelmann, 392-403. Opladen: Westdeutscher Verlag.

Lepsius, M. Rainer. 1997. Institutionalisierung Deinstitutionalisierung von Rationalitätskriterien. In Institutionenwandel, Hrsg. Gerhard Göhler, 57-69. Opladen: Westdeutscher Verlag.

Leuschner, Vincenz. 2016. Gewaltsituationen bei Schulamokläufen. In Situationen der Gewalt, Hrsg. Claudia Equit, Axel Groenemeyer, und Holger Schmidt, 304-322. Weinheim: Beltz Juventa.

Lindemann, Gesa. 2014. Weltzugänge. Weilerswist: Velbrück.

Lindemann, Gesa. 2015. Gewalt als soziologische Kategorie. Archiv für Rechts- und Sozialphilosophie 101(4):501-512.

Lindemann, Gesa. 2017. Verfahrensordnungen der Gewalt. Zeitschrift für Rechtssoziologie 37(1):57-87.

Lindemann, Gesa. 2018. Strukturnotwendige Kritik. Weilerswist: Velbrück.

Luhmann, Niklas. 1964. Funktionen und Folgen formaler Organisationen. Berlin: Duncker \& Humblot.

Luhmann, Niklas. 1984. Soziale Systeme. Frankfurt a. M.: Suhrkamp.

Luhmann, Niklas. 1992. Die Wissenschaft der Gesellschaft. Frankfurt a. M.: Suhrkamp.

Luhmann, Niklas. 2012. Macht im System. Berlin: Suhrkamp. 
Messmer, Heinz. 2003. Der soziale Konflikt: kommunikative Emergenz und systemische Reproduktion. Stuttgart: Lucius \& Lucius.

Meyer, John W., und Brian Rowan. 1977. Institutionalized orgasnizations. Formal structure as myth and ceremony. American Journal of Sociology 83(2):340-363.

Münch, Richard. 1984. Die Struktur der Moderne. Frankfurt a. M.: Suhrkamp.

Münch, Richard. 1991. Die Dialektik der Kommunikationsgesellschaft. Frankfurt a. M.: Suhrkamp.

Nassauer, Anne. 2015. Theoretische Überlegungen zur Entstehung von Gewalt in Protesten: Eine situative mechanismische Erklärung. Berliner Journal für Soziologie 25(4):491-518.

Nassauer, Anne. 2019. Situational breakdowns. Oxford: Oxford University Press.

Nedelmann, Birgitta. 1997. Gewaltsoziologie am Scheideweg. Die Auseinandersetzungen in der gegenwärtigen und Wege der künftigen Gewaltforschung. In Soziologie der Gewalt, Hrsg. Trutz von Trotha, 59-85. Opladen: Westdeutscher Verlag.

Nixon, Rob. 2011. Slow violence and the environmentalism of the poor. Cambridge: Harvard University Press.

Nungesser, Frithjof. 2017. Ein pleonastisches Oxymoron. Konstruktionsprobleme von Pierre Bourdieus Schlüsselkonzept der symbolischen Gewalt. Berliner Journal für Soziologie 27:7-33.

Nunner-Winkler, Gertrud. 2004. Überlegungen zum Gewaltbegriff. In Gewalt, Hrsg. Wilhelm Heitmeyer, Hans-Georg Soeffner, 21-61. Frankfurt a. M.: Suhrkamp.

Popitz, Heinrich. 1986. Phänomene der Macht. Tübingen: Mohr.

Ray, Larry. 2018. Violence \& society, 2. Aufl., London: Sage.

Reemtsma, Jan Philipp. 2009. Vertrauen und Gewalt. München: Pantheon.

Riekenberg, Michael. 2008. Auf dem Holzweg? Über Johan Galtungs Begriff der „strukturellen Gewalt“. Zeithistorische Forschungen 5:172-177.

Schimank, Uwe. 1985. Der mangelnde Akteurbezug systemtheoretischer Erklärungen gesellschaftlicher Differenzierung - Ein Diskussionsvorschlag. Zeitschrift für Soziologie 14(6):421-434.

Schimank, Uwe. 1988. Gesellschaftliche Teilsysteme als Akteurfiktionen. Kölner Zeitschrift für Soziologie und Sozialpsychologie 40(1):619-639.

Schimank, Uwe. 2000. Handeln und Strukturen. Weinheim: Juventa.

Schinkel, Willem. 2010. Aspects of violence. New York: Palgrave Macmillan.

Schmidt, Hajo. 2001. Krieg, Frieden und Gewalt im Denken Johan Galtungs. Leviathan 29(4):507-525.

Schroer, Markus. 2004. Gewalt ohne Gesicht. Zur Notwendigkeit einer umfassenden Gewaltanalyse. In Gewalt, Hrsg. Wilhelm Heitmeyer, Hans-Georg Soeffner, 151-173. Frankfurt a. M.: Suhrkamp.

Senghaas, Dieter. 1995. Frieden als Zivilisierungsprojekt. In Frieden als Zivilisierungsprojekt, Hrsg. Wolfgang R. Vogt, 37-55. Baden-Baden: Nomos.

Senghaas, Dieter. 1998. Zivilisierung wider Willen. Frankfurt a. M.: Suhrkamp.

Simmel, Georg. 1992. Soziologie. Frankfurt a. M.: Suhrkamp.

Sofsky, Wolfgang. 1996. Traktat über die Gewalt. Frankfurt a. M.: Fischer.

Sofsky, Wolfgang. 2002. Zeiten des Schreckens. Frankfurt a. M.: Fischer.

Staller, Mario S., und Swen Körner. 2020. Komplexe Gewaltprävention. Zum Umgang mit Gewalt auf individueller Ebene. Österreichische Zeitschrift für Soziologie 45(S1 1):157-174.

Sutterlüty, Ferdinand. 2015. Kollektive Gewalt und urbane Riots. Was erklärt die Situation? In Gewaltmassen, Hrsg. Axel T. Paul, Benjamin Schwalb, 231-256. Hamburg: Hamburger Edition.

Sutterlüty, Ferdinand. 2017. Fallstricke situationistischer Gewaltforschung. West End 14(2):139-155.

Swedberg, Richard. 2012. Theorizing in sociology and social scienece: turning to the context of discovery. Theory and Society 41(1):1-40.

Swedberg, Richard. 2014. From theory to theorizing. In Theorizing in social science. The context of discovery, Hrsg. Richard Swedberg, 1-28. Stanford: Stanford University Press.

Swedberg, Richard. 2016. Before theory comes theorizing or how to make social science more interesting. British Journal of Sociology 67(1):5-22.

Tranow, Ulf. 2018. Kontext als analytisches Konzept aus der Perspektive einer akteurszentrierten Soziologie. In Religion im Kontext, Hrsg. Annette Schnabel, Melanie Reddig, und Heidemarie Winkel, 21-41. Baden-Baden: Nomos.

von Trotha, Trutz. 1997. Zur Soziologie der Gewalt. In Soziologie der Gewalt, Hrsg. Trutz von Trotha, 9-56. Opladen: Westdeutscher Verlag.

Vereinte Nationen. 1948. Resolution der Generalversammlung 217 A (III). Allgemeine Erklärung der Menschenrechte. http://www.un.org/depts/german/menschenrechte/aemr.pdf;. Zugegriffen: 16. Sept. 2019.

Weber, Max. 1980. Wirtschaft und Gesellschaft, 5. Aufl., Tübingen: Mohr.

Wieviorka, Michel. 2003. Violence and the subject. Thesis Eleven 73:42-50.

Wieviorka, Michel. 2006. Die Gewalt. Hamburg: Hamburger Edition. 
Wieviorka, Michel. 2014. The sociological analysis of violence: new perspectives. The Sociological Review 62(2):50-64. 